When Does Group Information Influence the Automatic and Deliberate Judgment of

Individual Group Members?

\author{
Mayan Navon \\ Department of Psychology, Ben-Gurion University of the Negev, Beer-Sheva, Israel
}

Yoav Bar-Anan

School of Psychological Sciences, Tel-Aviv University, Israel

Correspondence author:

Mayan Navon, Department of Psychology, Ben-Gurion University of the Negev

E-mail: mayanna@post.bgu.ac.il

P. O. Box 653 Beer-Sheva, Israel

Tel: +972-50-2565900

Author note: This work was supported by the Israel Science Foundation [grant number 1684/21], awarded to Y. BarAnan. All materials, data, and pre-registrations of experiments are accessible at https://osf.io/zkejc/?view_only=59f0efaca05448d7b6ee8264cc188734 [MASKED LINK FOR REVIEW] 


\begin{abstract}
According to some impression formation theories, when people perceive an individual member of a social group, the information about the group is activated more spontaneously and easily than information specific to the individual. Therefore, judgment of individual group members might be more sensitive to group information (relatively to individuating information) the more automatic (fast, unintentional, and effortless) the judgment is. We tested this premise with a minimalistic impression formation paradigm that provided evaluative information about eight individuals and assigned them to two novel groups. In one group, three members behaved positively, and one member behaved negatively. In the other group, three members behaved negatively and one positively. In seven main experiments and 12 auxiliary experiments, we found a similar pattern with self-report measures and measures developed to capture automatic evaluation: the evaluation of the atypical group members was sensitive to the valence of the behaviors of the person (individuating information) more than to the valence of the typical behaviors in the group (group information). However, the relative effect of group information (vs. individuating information) was stronger on automatic than on deliberate judgment. This discrepancy increased when group membership was more salient upon judgment, and when participants belonged to one of the groups. Our findings suggest that although automatic judgment of individuals is sensitive to individuating information and group information, it is, inherently, more biased than deliberate judgment by group information. Further, under circumstances that are common in everyday life, that bias increases in automatic but not in deliberate judgment.
\end{abstract}

Keywords: social judgment, automatic evaluation, impression formation, individuation, prejudice 


\section{When Does Group Information Influence the Automatic and Deliberate Judgment of Individual Group Members?}

A prevalent notion in psychology is that information about social groups can bias people's judgment of individual group members despite motivation to base the judgment solely on the individual's own characteristics, rather than his or her group membership. The assumption is that a negative judgment of a stigmatized group might influence the judgment of the individual member, despite egalitarian motivations, due to automatic judgment processes (e.g., Devine, 1989; Dovidio et al., 1997; Fazio et al., 1995; Payne, 2001), that is, due to judgment processes that are relatively unintentional, uncontrolled, quick, or effortless (Bargh, 1994; Gawronski \& De Houwer, 2014).

Although prejudiced judgment (i.e., a judgment that is biased by information about social groups) of individual members of stigmatized groups is a common example for the possible consequences of stereotypes and stigma, most research on automatic judgment has focused on the judgment of social groups (e.g., Devine, 1989; Devine et al., 2002; Dovidio et al., 1997; Fazio et al., 1995; Greenwald et al., 1998; Kurdi et al., 2019; Nosek, 2005; Nosek et al., 2002; Phelps et al., 2000). The prevalent finding in that line of research suggests automatic preference for the dominant groups in one's society, and for one's ingroup, over non-dominant groups and outgroups (Bar-Anan et al., 2009; Dasgupta et al., 2000; Devine, 1989; Devine et al., 2002; Dovidio et al., 1997; Fazio et al., 1995; Greenwald et al., 1998; Kurdi et al., 2019; Nosek, 2005; Nosek et al., 2002; Nosek, Greenwald, et al., 2007; Ottaway et al., 2001; Phelps et al., 2000). Importantly, this automatic preference appears even when people report no preference between the groups when asked to make a deliberate judgment (e.g., Devine, 1989; Devine et al., 1991; Payne, 2001; Plant \& Devine, 1998).

Relatively to the vast research on the judgment of social groups, research on the deliberate and automatic judgment of individual members of social groups is scarce. Two sources of evaluative information may influence the judgment of individual group members: individuating information about the target individual, and information based on the social group of that individual. Knowledge based on group-membership (i.e., group information) is a schematic 
impression about all the members of that group. This type of knowledge links a certain trait or evaluative concept to all members of the group. Individuating information, on the other hand, includes the various traits, attributes, and actions that characterize that specific individual (Bodenhausen et al., 2012; Brewer, 1988; Fiske \& Neuberg, 1990). In the present research, we tested whether, relatively to non-automatic (deliberate) judgment, the automatic judgment of individual group members is inherently more sensitive to information about the group than to specific information about the individuals. We also examined what factors might moderate the influence of group information on the automatic and deliberate judgment of individual group members.

\section{Relevant Theories}

Early theories of person perception (e.g., Brewer, 1988; Fiske \& Neuberg, 1990) suggested that when perceivers judge individual members of known groups, they rely on group information, unless the person who makes the judgment is sufficiently motivated and has sufficient attentional resources to individuate the target individual. This hypothesis is based on the premise that perceivers are cognitive misers who seek to simplify the impression formation task under suboptimal conditions (Fiske \& Taylor, 1991). Using group information is often less effortful, compared with individuation (see reviews in Amodio \& Mendoza, 2010; Bodenhausen et al., 2012). For example, according to the Continuum Model (Fiske \& Neuberg, 1990), upon exposure to the individual, perceivers make an initial categorization based on salient apparent features and other quickly processed information. In turn, the categorization of the target individual leads to an automatic activation of thoughts, feelings, and behaviors, that were previously often activated upon encountering other members of that category (i.e., the social group). Any further processing and consideration of information other than the group information depends on the extent to which the target individual invokes motivations for further processing, and the extent to which the perceiver has enough attentional resources that can be devoted to the impression formation task. When motivation is low and attention is taxed or directed elsewhere, perceivers would base their 
impressions on the initial categorization. In contrast, if motivation is high and attention is devoted to the impression formation task, the influence of individuating information on judgment would increase.

The prominent theories about the relative effect of group and individuating information on judgment (Brewer, 1988; Fiske \& Neuberg, 1990) did not explicitly address automatic judgment. They hypothesized that influence of the group information would outweigh the influence of individuating information in most cases. However, the hypothesis that the processes that underlie the effect of group information are relatively less effortful than the processes that underlie the effect of individuating information suggests that the dominance of group information would be more prevalent in automatic than in deliberate judgments. As for the hypothesis that motivation to invest effort in the judgment can increase the effect of individuating information on judgment - that hypothesis seems more relevant to deliberate than to automatic judgment because the latter is less sensitive to effortful processes. Further, the motivation to avoid bias and use individuating information rather than group information might be prevalent because people tend to believe that individuals ought to be judged based on their own actions and traits rather than the actions of other people from the same group (Banaji \& Bhaskar, 2000; Thibault \& Walker, 1975). As a result, people might actively suppress the effect of group information on the judgment of the individuals while judging deliberately (Axt \& Lai, 2019; Ranganath \& Nosek, 2008). Such suppression efforts might be less effective on automatic judgment. Therefore, it seems that some prominent theories of person perception would suggest that deliberate judgment would be more likely to show sensitivity to individuating information than automatic judgment.

Another reason why group information might dominate the automatic judgment of individual group members is that the valence associated with of the social group might be activated automatically upon exposure to the face of the target individual. Specifically, theory suggests that, over time, certain facial features and configurations may acquire valence based on repeated pairing with the evaluation of the face's social group (Livingston \& Brewer, 2002). As a result of these 
pairings, the valence associated with the social group is activated automatically upon exposure to those facial features and configurations, independently of other sources of evaluative information. Thus, the presence of certain group characteristic features may trigger valence activation independently of the group information and the individuating information.

Is there any reason to assume that the automatic judgment of an individual member of a social group would not be particularly sensitive to information about the group? This possibility could be compatible with later theories of judgment and person perception. A later theory of person perception (Kunda \& Thagard, 1996; see also Monroe et al., 2018) has suggested that neither group information nor individuating information is the more prominent influence on person perception. Rather, the two types of information constrain each other. If the group information was observed prior to the individuating information, or was more strongly related to the target in one's memory, then it would dominate the judgment to a larger extent, compared with the individuating information. However, individuating information can also take primacy if it was observed first or has a stronger mental relation to the target. In other words, it is possible that the activation and application of information about the evaluative characteristics of the group is not always more unintentional, uncontrolled, or effortless than the activation of information about the evaluative characteristics of the individual.

More support for the possibility that evaluative information about the group might not have a privileged effect on automatic judgment can be derived from the distinction between acquisition and expression of evaluation. Automatic evaluation may reflect knowledge that has been encoded with much effort and deliberation. Therefore, even if ignoring group information and processing individuating information requires effort and time, the effortful information processing upon exposure to the information about an individual member of a social group may result with a mental evaluative representation that would be activated automatically, later, when encountering the same individual again. This possibility is consistent with many contemporary evaluation models, such as 
the associative and propositional model of evaluation (Gawronski \& Bodenhausen, 2018) and the propositional evaluation model (De Houwer, 2018).

In summary, we found theoretical basis for two opposing hypotheses. The possibility that group information would dominate automatic judgment of individual group members-despite knowledge of individuating characteristics of the target individual—is compatible with the premise that the processes that underlie the influence of group information on judgment are relatively effortless and might occur spontaneously when encountering the perceptual features of the social group. Further, the relative effect of group information versus individuating information might be stronger on automatic than deliberate evaluation because the processes that underlie the effect of individuating information on evaluation are effortful. The possibility that, upon deliberate evaluation, people are often motivated to invest that effort in order to avoid social bias further increases the likelihood of a discrepancy in the relative sensitivity of automatic vs. deliberate judgment to group vs. individuating information. On the other hand, later theories of person perception and judgment suggested that group information is not always more easily activated than individuating information, and that propositional reasoning processes at the stage of encoding the individuating information could equally influence automatic and deliberate judgment. Based on these assumptions, one might expect no discrepancy between deliberate and automatic judgment in their sensitivity to group vs. individuating information, and no dominance for the group information on deliberate or automatic judgment. Rather, the prevalent belief that people ought to be judged based on their own individual characteristics and not based on their group membership might lead to a dominance of individuating information in the automatic and deliberate judgment of individual group members.

\section{Relevant Evidence}

Research on deliberate judgment is based on the measurement of judgment with direct selfreport questionnaires. Some studies found support for the premise of early theories that group information is the default basis for deliberate judgment, and that relying on it is less effortful than 
relying on individuating information (Chun \& Kruglanski, 2006; Kruglanski \& Webster, 1991; Macrae et al., 1993, 1994). However, a comprehensive literature review and a meta-analysis (Kunda \& Thagard, 1996) suggested that group information dominates deliberate judgment mostly in the absence of individuating information, or when that information is ambiguous or non-diagnostic. Group information did not take primacy when the individuating information was unambiguous and diagnostic. Similar findings were obtained in a recent test of the Continuum Model (Monroe et al., 2018), in an experimental setup that did not induce the motivation to individuate. Overall, the available evidence suggests that, in contrast to early theorizing, group information does not serve as the default basis for deliberate judgment. Instead, perceivers use the most diagnostic information available upon judgment. When individuating information is more diagnostic than group information - perceivers rely more strongly on the individuating information in their judgments. What happens when judgment is relatively less deliberate? A few studies found that the relative effect of group information, in comparison to the effect of individuating information, is stronger, the less cognitive resources people have upon encoding the group and individuating information (e.g., Chun \& Kruglanski, 2006; Macrae et al., 1993). However, these studies are relevant to the effect of automatic processes during encoding rather than upon judgment. More relevant to the present question are studies that used indirect measures to capture automatic judgment. With these measures, the judgment is inferred from the participant's performance (usually reaction-time and accuracy) in different parts of the task, rather than from the participant's direct report (Gawronski \& De Houwer, 2014; Gawronski \& Hahn, 2019; Smith \& Ratliff, 2015). Experimental and correlational evidence suggests that these measures tap judgments that rely on processes that are relatively more automatic than the process that underlie self-reported judgment (Asendorpf et al., 2002; DeSteno et al., 2004; Devine et al., 2002; Egloff \& Schmukle, 2002; Fazio et al., 1986; Hermans et al., 2000; Kim, 2003a; Klauer \& Teige-Mocigemba, 2007; Nosek, Greenwald, et al., 2007; Schmitz et al., 2013; Steffens, 2004a; Wittenbrink et al., 2001). For example, in comparison to self-reported evaluation, indirect measures are relatively more immune 
to the faking of the measurement outcome, compared with self-report (Asendorpf et al., 2002; Egloff \& Schmukle, 2002; Kim, 2003b; Nosek, Smyth, et al., 2007; Steffens, 2004b), capture efficient processes that require less cognitive effort (e.g., Devine et al., 2002; Hermans et al., 2000; Ito et al., 2015; Klauer \& Teige-Mocigemba, 2007; Schmitz et al., 2013), and are better in predicting behaviors performed under cognitive load than of behaviors performed without a load (e.g., Friese et al., 2008; Hofmann et al., 2007, 2008; Wiers et al., 2009).

Yet, indirect measures of evaluation are not perfectly valid because they sometimes capture processes that might be unrelated to judgment (Brown-Iannuzzi et al., 2019; Olson \& Fazio, 2004; Uhlmann et al., 2006) or to automaticity (Greenwald \& Nosek, 2001; McFarland \& Crouch, 2002; Mierke \& Klauer, 2003; Steffens, 2004a; Teige-Mocigemba \& Klauer, 2008; Unkelbach \& Fiedler, 2020). Therefore, evidence collected with such indirect measures is only suggestive, especially if not replicated with various, different indirect measures. Any such evidence could be challenged in the future with specific alternative accounts that attribute the observed effects to specific nonevaluative or non-automatic factors. Currently, however, reliance on converging evidence from multiple indirect measures is the best available research tool for investigating automatic judgment (see discussions in Corneille \& Hütter, 2020; Navon et al., 2021; Vianello \& Bar-Anan, 2021). Investigations of the automatic judgment of individual group members using the most commonly employed indirect measure of judgment (Nosek et al., 2011), the Implicit Association Test (IAT; Greenwald et al., 1998), suggested that, similar to deliberate judgment, group information dominates automatic judgment when individuating information is ambiguous or absent (e.g., Cao \& Banaji, 2016; Castelli et al., 2004; Ranganath \& Nosek, 2008). Only a few studies used indirect evaluation measures to examine the judgment of individual targets when both group information and diagnostic individuating information are available. These studies used fictitious (Cao \& Banaji, 2016; Rubinstein et al., 2018) or well-known (Navon et al., 2021) target individuals from existing social groups (race, age, and gender groups). For the fictitious targets, participants read diagnostic individuating information about them prior to the judgment task. For well-known 
targets (famous musicians and actors), participants accrued the individuating information long before they came to the lab.

In one set of studies (Cao \& Banaji, 2016), the IAT measured the relation between a specific man and a specific woman with a stereotypically-feminine occupation and stereotypicallymasculine occupation, after the participants read that the actual occupation of these particular man and woman was the counter-stereotypic occupation (this was the diagnostic individuating information). That study found that the diagnostic individuating information dominated the selfreported judgment, but had much smaller influence on IAT scores: participants still showed a score that is consistent with the stereotype, but to a lesser extent than before they read the individuating information. Evidence for a similar discrepancy between automatic and deliberate evaluation was found in a set of studies (Navon et al., 2021) that used the IAT and the Evaluative Priming Task (EPT; Fazio et al., 1995) to measure the automatic evaluation of pairs of famous people: one person belonged to a stigmatized group (Black people or old people) and the other person belonged to a dominant group (White people or young people). Participants usually reported a preference for the individual member of the stigmatized group but the indirect measures suggested an automatic preference for the individual member of the dominant group.

In another set of studies (Rubinstein et al., 2018), participants judged the intelligence of a Black person and a White person, after reading individuating information that varied in its diagnosticity (from low to high). Group information (the stereotype that White people are more intelligent than Black people) determined the individual's self-reported judgment and IAT scores under low diagnosticity, but when the individuating information was highly diagnostic this influence was eliminated for self-report and substantially reduced for IAT scores.

In summary, the evidence thus far suggests that in the absence of diagnostic individuating information, group information serves as the source of evaluative information perceivers use in their deliberate and automatic judgment of target individuals. When diagnostic individuating information about the target individuals is available, there is an increase in the influence of individuating 
information on both deliberate and automatic judgment. However, the evidence suggests a discrepancy: when both group information and diagnostic individuating information are available, automatic judgment is more sensitive than deliberate judgment to group information.

\section{Is There a Basic Tendency to rely on Information about the Group in Automatic Judgment?}

The findings summarized in the previous section suggest that group information might have a larger influence on automatic than on deliberate judgment of individual group members. In the present research, we sought to extend research on this question in a paradigm that provided us full control on the group information and the individuating information. We designed that paradigm to provide a clean comparison between group information and personal characteristics in their effect on judgment. In previous research, all the individual targets belonged to existing social groups that participants have already known (specifically, Black/White people, women/men, and old/young people). The researchers had no specific knowledge on the information that each participant accumulated over the years regarding the social groups, and the experience that each participant had with members of these groups. The existing groups that previous research used might influence judgment due to reasons specific for these well-known groups and categories (age, race, and gender), rather than due to basic properties of group information and the effect of these properties on automatic and deliberate judgment. Further, factors such as the perceiver's motivations and extrapersonal associations may contribute to the judgment of the target individual. For instance, group identification and the motivation to maintain positive self-esteem are known to result in intergroup comparisons that favor members of the ingroup over outgroup members (Oakes \& Turner, 1980). Additionally, evidence suggests that the automatic judgment of existing social groups might sometimes reflect associations between the group and concepts that do not reflect the perceiver's actual judgment (Brown-Iannuzzi et al., 2019; Olson \& Fazio, 2004; Uhlmann et al., 2006), such as an association between Black people and the concepts poor and oppressed, rather than disliked or unfavorable (Uhlmann et al., 2006). 
Moreover, in studies that use fictitious target individuals from existing social groups (Cao \& Banaji, 2016; Rubinstein et al., 2018), there was an inherent asymmetry between the two sources of information: while the participants read the individuating information about these individuals in the lab, immediately before the judgment task, they have been exposed to the group information much earlier, over the course of their lives. Therefore, the group information might have had an advantage because it has been extensively used and potentially rehearsed in the past, and not because it was information about the group. Research that did use novel social groups (Ranganath \& Nosek, 2008) has not provided diagnostic individuating information about the target individuals. Therefore, there is currently no evidence pertaining to the present research question: is there an inherent tendency for automatic judgment of individual group members to rely on information about the group when individuating information is available?

\section{The Present Research}

The purpose of the present research was to test the effect of group information on the automatic judgment of an individual group member, when there is ample relevant information specific to the individual. We presented to the participants novel fictitious social groups in order to examine whether the mere fact that an individual belongs to a group would lead to an effect of knowledge about the group on the automatic evaluation of that individual, even if the perceiver had enough individuating information about the target individual. We took care to provide the group information in the same method that we provided the individuating information. The individuating information was the typical behavior of an individual group member. The group information was the typical behaviors of each of the other group members. We exposed participants to information about the group and the target individual at the same time, thus reducing the possibility that group information would have the privilege of long-existing information that have been accumulated long before the experiment, unlike research that used pre-existing social groups. Further, the use of novel social groups eliminated the possibility that various motivations, beliefs, and non-evaluative associations pertaining to the group would influence the participants' response. Finally, while prior 
research on this subject focused mainly on trait-judgments (i.e., stereotypes; Cao \& Banaji, 2016; Rubinstein et al., 2018), we investigated evaluation (prejudice).

In our basic paradigm, we tried to provide the minimal information needed for forming an accurate evaluation of the whole group and of each of the group's individual members. Our purpose was to test whether these conditions are sufficient for inducing prejudice in the automatic evaluation of the individual members of the group. We reasoned that if we find an effect of group information on automatic evaluation, even when the group has been previously unknown, it would rule out the possibility that previous evidence of such prejudice (e.g., Navon et al., 2021) reflects the effect of intergroup conflict (i.e., ingroup favoritism) or the effect of differences in the amount or type of information that people have accumulated about groups over the years (relatively to the information about the target individual). On the other hand, if we do not find much evidence for a dominance of group information on automatic evaluation, or for discrepancy between deliberate and automatic evaluation in their sensitivity for group information - it would suggest that automatic evaluation does not privilege group information per se. In that case, perhaps specific features that were included in previous research with pre-existing groups were responsible for the effect of group information on automatic judgment.

The experimental paradigm that we developed for this research is useful for testing possible moderators of the relative effect of group information and individuating information on the evaluation of individual group members. In the second part of this manuscript, we report experiments that tested whether adding to our minimalistic paradigm features that might characterize social groups based on race, gender, and age (the groups used in previous relevant research) would increase the effect of group information on automatic judgment. Specifically, the factors that we tested were the amount of information provided about the group, the salience of group-membership upon judgment of the individual, and motivational relevance (i.e., whether the participant is a member of one of the groups). Most participants have accumulated much information about each of the basic social groups used in previous research (race, age, and gender), 
and that information might be extremely skewed toward certain stereotypes and prejudice. Race, age, and gender are also quite salient in most people's mind and are categorized automatically and quickly (Ito \& Urland, 2003, 2005). Finally, most participants have personal relation to race, age, and gender, and that could activate motivations, such as ingroup favoritism. We experimentally manipulated each of these three factors to examine whether it would moderate the effect of group information on the judgment of individual group members.

\section{Overview of the Present Experiments}

Across all experiments, we used a basic paradigm that provided participants evaluative information about the members of two fictitious social groups. Participants read typical behaviors of four members of each group (different behaviors for each member). Each individual was characterized by either positive or negative behaviors. In one group, three members were portrayed positively and one negatively. In the other group, three members were negative and one was positive. The group information was the prevalent valence of the behaviors of members of each group. The individuating information was the valence of the behaviors that characterized each individual member. Afterwards, participants evaluated a pair of target individuals, one from each group. Half of the participants evaluated a pair of targets who were typical of their group: group members who had the same valence as most of the individuals in their group. The rest of the participants evaluated an atypical pair of targets: the group members whose characteristic behaviors were of valence opposite to the valence of the characteristic behaviors of the other three group members. In other words, participants either judged two target individuals who were typical of their group or the two atypical targets. If we find that in both conditions, participants favored the positively portrayed individual over the negatively portrayed individual to a similar extentregardless of the valence of the majority of their social group - that would suggest that participants relied solely on the individuating information. In contrast, any reduction in the preference for the positively-portrayed target over the negatively-portrayed target in the atypical-targets condition 
(i.e., when the group information is opposite to the individuating information) relatively to the typical-targets condition would reflect an effect of the group information on evaluation.

Like all previous research on the automatic judgment of individual group members, we measured deliberate evaluation with self-reported questionnaires and automatic evaluation with indirect measures that were developed to measure evaluative process that are relatively more automatic and less deliberate than the evaluative processes that influence self-reported evaluation (Gawronski \& De Houwer, 2014; Smith \& Ratliff, 2015). As we already mentioned, the current evidence about the validity of these measures is equivocal. Yet, currently, reliance on converging evidence from multiple indirect measures is the best available research tool for investigating automatic judgment (see discussions in Corneille \& Hütter, 2020; Gawronski, De Houwer, \& Sherman, 2020; Navon et al., in press; Vianello \& Bar-Anan, 2021). In the present research, we used four indirect measures that were developed to measure automatic judgment. No published work provided evidence for an alternative account to a supposed finding about automatic judgment that was corroborated by more than one indirect measure.

In Experiments 1-3, we used our basic paradigm to test participants' reliance on individuating information and group information in their deliberate and automatic evaluations of individual group members. For generalization beyond a specific supposed measure of automatic evaluation, each experiment used a different indirect measure of evaluation. We then meta-analyzed the results from Experiments 1-3 and a number of auxiliary experiments that used the same paradigm. To preface our results, individuating information had a large effect on the automatic and the deliberate evaluation of the target individuals, while group information had only a small effect on automatic evaluation, and an even a smaller effect on deliberate evaluation. Experiments S6 and S7 (online supplement) successfully verified that our paradigm does capture the effect of group information on the evaluation of target individuals, when there is no relevant individuating information about the targets, as found in previous research (Cao \& Banaji, 2016; Castelli et al., 2004; Kunda \& Sherman-Williams, 1993; Ranganath \& Nosek, 2008). 
In Experiments 4-7, we examined several possible moderators for the effects of group information and individuating information on evaluation by factors that might mimic the characteristics of existing social groups. We tested whether the relative effect of group information on the automatic and deliberate evaluation of target individuals increases when we increase the amount of information about the group (Experiment 4), when group membership is more salient upon judgment (Experiment 5), and when motivational relevance is high (when the perceiver belongs to one of the groups, Experiments 6-7).

\section{Experiment 1: The Basic paradigm}

Experiment 1 tested the effects of individuating information and group information on deliberate and automatic evaluation, measured with the IAT.

\section{Method}

We report all data exclusions, manipulations, and measures, and how we determined our sample sizes on each of the experiments. The decision to stop collecting data did not depend on the obtained results. The materials, data, and pre-registrations of the experiments are accessible at https://osf.io/zkejc/?view_only=59f0efaca05448d7b6ee8264cc188734 [MASKED LINK FOR REVIEW].

\section{Participants}

Participants in all experiments were volunteers at the Project Implicit website (implicit.harvard.edu; Nosek, 2005). In each of our experiments, we planned to recruit enough participants to achieve at least $80 \%$ power for an effect size of a reasonable size in social psychology (Cohen's $d=0.25$, Pearson $r=.25, \eta_{p}^{2}=.02$ ). The pre-registration documents provide further details about our power calculations for each experiment. Table 1 presents, for each experiment, the number of participants who (1) started the experiment, (2) completed the experiment, (3) were excluded from the experiment, and (4) were included in the analyses (along with their demographic information). We excluded participants who had $>10 \%$ fast trials in the IAT 
(RT< 300 ms; see Greenwald et al., 2003). All studies were approved by the ethics committee of the Psychology department at ***[MASKED for the review] University.

\section{Materials}

Group names. We generated a list of eight novel group names using a letter randomizer (http://www.dave-reed.com/Nifty/randSeq.html). The resulting group names were: Ovoomps, Beliks, Noonnips, Baunifs, Coawaqs, Glags, Qainfins, Roskipps. We additionally added two groupnames used in previously published studies (e.g., Ranganath \& Nosek, 2008): Laapians and Niffians. For each participant, we randomly selected two group names from this set.

Behaviors list. We used a list of 23 positive behaviors and 23 negative behaviors (see online supplement), collected from previous impression formation research (Bar-Anan \& Nosek, 2012; Ranganath \& Nosek, 2008). For each participant, the program randomly selected 16 positive and 16 negative behaviors, and randomly assigned the positive behaviors to the four positively portrayed target individuals (three in the "positive" group and one in the "negative" group) and the negative behaviors to the four negatively portrayed targets (three in the "negative" group and one in the "positive" group).

Face dataset. We generated a pool of 17 male face identities from a subset of the neutral faces from the Karolinska Directed Emotional Faces database (KDEF, Lundqvist et al., 1998). Each face identity had six photos, showing that face in different poses (e.g., direct gaze, posing left). We slightly brightened all images using the Paint.Net image editing software (Paint.Net, version 4.2.13, 2020). Using the same software, we colored the skin of the faces in each photo, once in green and once in purple. These skin colors served as visual cues that differentiated between the two social groups. For each participant, we randomly selected eight face identities, four with each skin color, as the members of the two groups. 
Table 1

Experiments 1-7: Details about the participants

\begin{tabular}{llllllll}
\hline Experiment & Started & Finished & Excluded & Included & Mean Age (SD) & Sex $^{\mathrm{b}}$ & Race $^{\mathrm{c}}$ \\
\hline 1 & 1,252 & 618 & 18 & 600 & $34.34(14.32)$ & $66.66 \%$ & $75.33 \%$ \\
2 & 1,233 & 586 & 10 & 576 & $33.66(13.63)$ & $66.14 \%$ & $71.00 \%$ \\
3 & 1,231 & 603 & 88 & 515 & $32.89(13.62)$ & $67.76 \%$ & $70.48 \%$ \\
4 & 1,010 & 615 & 6 & 601 & $38.67(15.69)$ & $69.55 \%$ & $73.38 \%$ \\
5 & 3,313 & 1,984 & 102 & 1,882 & $34.36(14.20)$ & $61.26 \%$ & $70.61 \%$ \\
6 & 1,451 & 972 & 38 & 934 & $36.70(15.71)$ & $64.03 \%$ & $67.99 \%$ \\
7 & 1,481 & 950 & 27 & 923 & $37.19(15.48)$ & $63.92 \%$ & $70.31 \%$
\end{tabular}

Note. Demographics pertain to the "included" samples. ${ }^{\mathrm{b}}$ Proportion of females. ${ }^{\mathrm{c}}$ Proportion of White participants. 


\section{Procedure}

We describe the procedure in detail only in Experiment 1 because it was nearly identical in the other experiments. The experiment had two main parts: induction and evaluation. The online Supplementary Materials present a detailed description of all task instructions ${ }^{1}$.

\section{Induction.}

Before the task started, we showed the participants the faces of the four people who belonged to each of two groups (eight faces in total) together on one screen, with the following instructions:

In the first part in the study, you will meet individuals belonging to two groups: the [NAME OF GROUP 1] and the [NAME OF GROUP 2]. All [NAME OF GROUP 1] have a purple skin tone, whereas all [NAME OF GROUP 2] have a green skin tone. Your task is to form an impression about each group. Later, we will ask you what you feel about them.

Here are the groups and their members. Please take a couple of minutes to learn which faces belong to each group:" (the faces appeared below this text).

In the next screen, participants read the following instructions:

Next, you will meet the individuals belonging to the [NAME OF GROUP 1] and the [NAME OF GROUP 2] and learn about their typical behaviors. Remember, your task is to form an impression of each group. Later, we will ask you what you feel about them. Each screen, showing an individual's face, group membership, and behavior will appear for 5 seconds, and then the next screen will appear automatically. You will not have to press any key. Please press SPACE to start the task.

These instructions were designed to focus participants on the fact that the individuals belonged to two groups, and that each group was characterized with predominantly positive or negative behaviors. We planned to use subtler instructions if we find strong effect of the group

\footnotetext{
${ }^{1}$ The pre-registered method included a face-sorting task (to strengthen the link between the individuals and their groups) that we eventually did not include in the experiment, to keep it short.
} 
information in the basic paradigm, but we did not find such an effect. On each trial of the induction task, participants viewed the group's name (e.g., Group: Baunifs), a single group member's face, and a single behavior, either positive or negative. The next trial began after five seconds.

Participants completed four blocks (32 trials in total), each showing each group member once, with a behavior. That is, overall, participants read four behaviors of each group member. On each of the task's blocks, participants first viewed all members of a certain group in four trials, and then viewed all the members of the other group in another four trials. The presentation order of the two groups was randomized between participants. Within each group's presentation on each block, the presentation order of the group members - including the atypical member - was randomized between participants.

Following the four blocks, participants completed a final reminder block. On this block, participants viewed each group member with all his behaviors (that appeared in Blocks 1-4) presented on one screen. Participants were instructed to read all the behaviors, and then press the Space key to view the next group member. This block consisted of eight trials (four for each group), one for each of the group member. The presentation order of the two groups' trials was randomized between participants. Within each group's presentation in the last block, the presentation order of the typical targets was randomized between participants, while the atypical target always appeared last. This was done to encourage participants to attend to the atypical target and notice that he is opposite from his group. The instructions for the reminder block were:

We know that it is difficult to process all the information we presented to you after reading it only once. To make it easier, we will show you the eight individuals and their behaviors again. This time, you will see each individual with all his behaviors at once on one screen. For each individual, please read all the behaviors. After reading each person's behaviors, please press SPACE to see the next individual. Your task is still the same: Please try to form an impression of each group. Press SPACE to start. 
Each group consisted of four group members. One group was mostly positive: the four characteristic behaviors of three members (typical targets) were positive, while the behaviors of the fourth group member were negative (atypical target). The other group was opposite in valence: three group members had negative behaviors (typical members), while one group member had positive behaviors (atypical target).

Evaluation. Following the induction part, participants continued to the evaluation part. They evaluated a pair of group members, one from each group. Half of the participants evaluated a pair of individuals who were typical of their group (individuals whose behaviors were of the same valence as the behaviors of two other individuals in their group), and half evaluated a pair of individuals who were the atypical member of their group (individuals whose behaviors were of the opposite valence of the behaviors of the other three individuals in their group). Participants completed the self-reported evaluation measure and the IAT, in a random order.

Implicit Association Test (IAT; Greenwald et al., 1998). The IAT is a classification task that has been widely used as a measure of automatic judgment. In this task, participants classify items (photos and/or words) to categories, using two key responses. The association between the categories is estimated based on the ease of classification when two categories share the same response key. The two target categories were the two target individuals. The label of each target category was the face of the target individual. The items of each target category were five photos of the person's face, facing different directions. The labels of the attribute categories were Pleasant words (items: positive, pleasant, good, outstanding, beautiful, magnificent, marvelous, excellent, appealing, delightful) and Unpleasant words (items: negative, bad, horrible, miserable, hideous, dreadful, painful, repulsive, awful, ugly). We used the standard seven blocks design (Nosek, Greenwald, et al., 2007), with a random assignment of the first pairing condition in the task (i.e., whether the positively portrayed target individual shared a key with Good words or with Bad words). Before the task started, participants were instructed as follows: "Two of the categories in 
the task will be two individual members from the [NAME OF GROUP 1] and [NAME OF GROUP

2] groups. Here are the individuals:" Images of the two targets appeared below.

For each participant, we computed an IAT D score (using the D600 algorithm, Greenwald et al., 2003), such that scores above zero indicated a preference for the positively portrayed target over the negatively portrayed target (i.e., faster responses when the positive target shared a key with Pleasant words, and the negative target shared a key with Unpleasant words than when the key assignment was the opposite $)^{2}$.

Self-reported evaluation (adapted from Moran et al., 2017). Participants were instructed as follows:

Next, we will ask you about your feelings toward two individual members from the [NAME OF GROUP 1] and [NAME OF GROUP 2] groups. Here are the individuals: [images of the two targets appeared below]. There will be 6 questions in total. There are no correct or incorrect answers to the questions. We are interested in your genuine thoughts and feelings. Then, about each of the two targets, participants answered three questions in the format of How likable [trustworthy, friendly] do you think that this man is? The face of the relevant target individual appeared next to the question's text (thus, the object of evaluation was the same across the self-report measure and the IAT measure: the target individuals, represented by their faces). The response options ranged from 1 (Extremely unlikeable [untrustworthy; unfriendly]) to 9 (Extremely likeable [trustworthy; friendly]). The order of the six questions (three for each of the two group members) was randomized for each participant. To compute a self-reported evaluation score, we averaged the responses to the three questions regarding each group member. For a self-reported preference score, we subtracted the evaluation of the negatively portrayed target from the evaluation of the positively portrayed target.

\footnotetext{
${ }^{2}$ The pre-registration indicates that we would compute a modified IAT score, with separate scores for each pairing condition. We thought that it would simplify the analysis, but eventually decided that it would be more confusing than using the standard IAT score. The results with the modified scores appear in the online Supplementary Materials and are equivalent (and, hence, lead to exactly the same conclusions) as the results we report here.
} 
Following the induction task and the evaluation measures, participants completed a selfreport measure of memory for the pair of targets' valence, followed by a self-report measure of the groups' evaluation. The latter measure served as a manipulation check of the induction task.

Self-reported group evaluation. Participants responded to the same three questions of the self-reported evaluation measure (in a random order), but this time regarding the average group member (e.g., How likable do you think that the average Baunif is?). We used the same procedure described above to compute a preference score for the positive group (positive behaviors characterized three of the four individuals of that group) over the negative group.

The online Supplementary Materials ([MASKED LINK FOR REVIEW] https://osf.io/tpws3/?view_only=9234a16cdc8747f6868b3da87a9c9705) include several measures of participants' memory for the provided information (i.e., individuating information and group information) that were added to each of the experiments for exploratory reasons. The full description of these measures, their results, and an overview of conclusions from all the memory results (with no valuable information regarding the present research questions) are detailed in the supplement.

\section{Results}

\section{Manipulation check: Self-reported group evaluation}

Table 2 presents the descriptive statistics and reliability (internal consistency) of each measure. To verify that participants attended to the behavioral information and the group membership of the individuals, we tested whether the information influenced self-reported evaluation of the groups. Participants reported a preference for the group that included three positively portrayed individuals over the group that included three negatively portrayed individuals $\left(M_{\text {diff }}=1.70, S D_{\text {diff }}=3.37\right), t(599)=12.33, p<.001, d=0.50, B F_{10}>1,000$.

\section{Individuation and group effects}

Self-reported evaluation. A 2 (target's valence: positive, negative) x 2 (targets' typicality relatively to their group: typical, atypical) ANOVA on the evaluation scores of the two targets, 
found a main effect of target's valence, $F(1,598)=568.78, p<.001, \eta^{2}{ }_{p}=.49$, that suggested a preference for the positively portrayed target over the negatively portrayed targets (see Table 2). There was no main effect of targets' typicality, and no interaction, $p s>.10$. In other words, Experiment 1 found no evidence for the effect of group information on the self-reported evaluation of the target individuals; only strong evidence for the effect of the individuating information.

Indirectly-measured evaluation (IAT). The scores suggested a preference for the positively portrayed target over the negatively portrayed targets, when those targets were typical of their groups $(M=0.22, S D=0.41), t(316)=9.56, p<.001, d=0.54, B F_{10}>1,000$, and when they were atypical of their groups $(M=0.14, S D=0.44), t(282)=5.43, p<.001, d=0.32, B F_{10}>1,000$. An independent samples t-test on the IAT scores as a function of the targets' typicality condition found only a small effect of targets' typicality, $t(598)=2.26, p=.024, d=0.18, B F_{10}=1.09$. In other words, we found only weak evidence for the effect of group information on the automatic evaluation of the target individuals. The fact that both conditions presented positive IAT scores suggests that the effect of individuating information on the evaluation was larger than the effect of the group information.

The online Supplementary Materials include a report of the results from tests of the moderation of the primary effects by various order variables, with no significant contribution to the research questions.

\section{Discussion}

Experiment 1 did not find good evidence that automatic evaluation prioritizes group information above information specific to the individual member of the group. The individuating information strongly influenced both the deliberate and the automatic evaluation of the targets, as measured with self-report and the IAT respectively. However, we found some evidence that group information influenced evaluation only for automatic and not for deliberate evaluation, compatible with the hypothesis that group information has a larger effect on automatic than on deliberate evaluation. Still that influence (i.e., of group information on automatic evaluation) was much 
smaller than that of individuating information, suggesting individuating information played the more central role in participants' deliberate and automatic impressions. To further improve the knowledge about of these effects, Experiments 2 and 3 replicated Experiment 1 with different indirect measures that were developed to measure automatic evaluation.

\section{Experiments 2-3: Replication with Other Indirect Measures of Evaluation}

Although there is some evidence that each of the measures that were developed to measure automatic evaluation is sensitive to automatic evaluation, there is also some evidence that the other factors sometimes influence each of them (e.g., Rothermund \& Wentura, 2001, 2004). Further, it is not well-established what features of automaticity characterize the evaluation captured by each of the available measures. Therefore, a successful conceptual replication of studies with several supposed measures of automatic evaluation can increase the confidence in the possibility that the finding indeed pertains to automatic evaluation. Different results with different measures would provide data about the distinction between the measures (Vianello \& Bar-Anan, 2021). Following this logic, we replicated Experiment 1 with different supposed measures of automatic evaluation: The Sorting Paired Features task (SPF, Bar-Anan et al., 2009) in Experiment 2, and the Affect Misattribution Procedure (AMP, Payne et al., 2005) in Experiment 3 (we used a fourth supposed measure of automatic evaluation in Experiment 7).

\section{Methods}

\section{Participants}

The details about the participants appear in Table 1 . We excluded participants who had $>10 \%$ fast trials in the SPF (RT < 300 ms; Bar-Anan et al., 2009) and participants who had more than $95 \%$ or less than $5 \%$ 'pleasant' responses in the AMP (Moran et al., 2017). Additionally, for participants who completed the AMP (Experiment 3), we excluded participants who reported being able to read Chinese. 


\section{Measures and materials}

All materials and measures were the same as in Experiment 1, except for the indirect measure. Instead of the IAT, participants completed the SPF (Experiment 2) or AMP (Experiment $3)$.

Sorting Paired Features (SPF). The SPF (Bar-Anan et al., 2009) is a classification task. The unique property of the SPF is that it measures in a single block the four associations measured across different IAT blocks. For that reason, it eliminates block-order effects that are sometimes witnessed on the IAT. On each trial, the participant is required to sort pairs of stimuli (e.g., the Baunif's face + Ugly) to one of four possible category pairs (the Baunif's face + Pleasant, the Baunif's face + Unpleasant, the Lapian's face + Pleasant, the Lapian's face + Unpleasant), appearing in the four corners of the screen. The SPF consisted of three blocks, with ten trials for each of the four category + attribute combination on each block. The items and categories were the same as in the IAT in Experiment 1. Prior to the score computation, we excluded participants with > $10 \%$ fast trials $(\mathrm{RT}<300 \mathrm{~ms}$ ), and trials faster than $400 \mathrm{~ms}$ or slower than $10,000 \mathrm{~ms}$ (Bar-Anan et al., 2009). We computed a preference D score as in Bar-Anan et al. (2009), so that scores larger than zero reflect a preference toward the group member who behaved positively.

Affect Misattribution Procedure (AMP). The AMP (Payne et al., 2005) is a sequential priming task. Each trial begins with the presentation of a prime stimulus (e.g., the Baunif's face), followed by a target stimulus (a Chinese character), followed by a mask stimulus. The participants' task was to judge whether the target stimulus is more pleasant or more unpleasant than the average Chinese character (the judgment labels were Pleasant and Unpleasant). They were instructed to ignore the first item (the prime) and concentrate on the second item (target). The primes were the faces of the two target individuals (as in the IAT and SPF) and were presented for $100 \mathrm{~ms}$, followed by a post-prime blank screen presented for $100 \mathrm{~ms}$, a target stimulus presented for $100 \mathrm{~ms}$, and a mask presented until response. The intertrial interval was $250 \mathrm{~ms}$. The AMP included three blocks of 40 trials (20 trials for each prime category). Before the main task started, participants completed 
three practice trials with neutral prime stimuli (e.g., pictures of a lamp and umbrella). On these trials, the target stimuli were presented for $300 \mathrm{~ms}$. The preference score was the result of the subtraction of the rate of pleasant responses in trials in which the negatively portrayed individual was the prime stimulus from the rate of pleasant responses in trials in which the positively portrayed individual was the prime stimulus.

\section{Results}

Table 2

Experiments 1-3: Descriptive statistics for the preferences between the group members

\begin{tabular}{lllllll}
\hline Experiment & Targets' typicality & Preference & $N$ & Mean & $S D$ & $\alpha^{\mathrm{a}}$ \\
\hline 1 & Typical pair & Deliberate & 317 & 3.27 & 2.92 & $0.82,0.87$ \\
& IAT & 317 & 0.22 & 0.41 & 0.81 \\
& Atypical pair & Group & 317 & 2.01 & 3.06 & $0.83,0.84$ \\
& Deliberate & 283 & 3.02 & 3.54 & $0.85,0.92$ \\
& IAT & 283 & 0.14 & 0.44 & 0.83 \\
& Typical pair & Group & 283 & 1.35 & 0.22 & $0.86,0.90$ \\
& Deliberate & 293 & 3.48 & 2.65 & $0.82,0.86$ \\
& SPF & 293 & 0.16 & 0.50 & 0.40 \\
& Atypical pair & Group & 293 & 2.44 & 0.17 & $0.77,0.85$ \\
& Deliberate & 283 & 3.29 & 3.33 & $0.89,0.91$ \\
& SPF & 283 & 0.11 & 0.49 & 0.34 \\
& Typical pair & Group & 283 & 1.99 & 3.35 & $0.82,0.84$ \\
& Deliberate & 264 & 3.80 & 2.43 & $0.83,0.85$ \\
& AMP & 264 & 0.14 & 0.34 & 0.94 \\
& Atypical pair & Group & 264 & 2.35 & 2.80 & $0.70,0.80$ \\
& Geliberate & 251 & 3.41 & 3.04 & $0.86,0.90$ \\
& AMP & 251 & 0.10 & 0.33 & 0.94 \\
& & 251 & 1.72 & 3.25 & $0.82,0.85$
\end{tabular}

Note. In all measures, positive values reflect a preference for the positive target or group (Group measure) over the negative target or group (Group measure). ${ }^{a}$ For the deliberate evaluations and group evaluations, the left value refers to the positive target (group) items, and the right value refers to the negative target (group) items. 


\section{Manipulation check - Group evaluation}

Table 2 presents the descriptive statistics and reliability (internal consistency) of each measure. In both experiments, participants correctly learned the valence of each group, as they evaluated the positive group more positively relative to the negative group. Experiment 2: $M_{\text {diff }}=$ 2.22, $S D_{\text {diff }}=3.17, t(575)=16.80, p<.001, d=0.70, B F_{10}>1,000$. Experiment $3: M_{\text {diff }}=2.04$, $S D_{\text {diff }}=3.04, t(514)=15.24, p<.001, d=0.67, B F_{10}>1,000$.

\section{Individuation and group effects}

Self-reported evaluation. As in Experiment 1, we conducted a 2 (target's valence: positive, negative) x 2 (targets' typicality relatively to their group: typical, atypical) ANOVA on the evaluation scores of the two targets. As in Experiment 1, there was a main effect of target's valence, Experiment 2: $F(1,574)=729.56, p<.001, \eta^{2} p=.56$, Experiment $3: F(1,513)=886.35, p<$ $.001, \eta_{p}^{2}=.62$, and no main effect of targets' typicality, or interaction, $\eta_{p s}^{2}<.56, p s>.10$. Thus, as in Experiment 1, we found no evidence for the effect of group information on the deliberate evaluation of the target individuals.

Indirectly-measured evaluation (SPF, AMP). In both experiments, the scores reflected a preference for the positively portrayed target over the negatively portrayed targets, when those targets were typical of their groups, Experiment 2: $M=0.16, S D=0.50, t(292)=5.61, p<.001, d=$ 0.33, $B F_{10}>1,000$, Experiment 3: $M=0.14, S D=0.34, t(263)=6.77, p<.001, d=0.42, B F_{10}>$ 1,000 , and when they were atypical of their groups, Experiment $2: M=0.11, S D=0.49, t(282)=$ 3.67, $p<.001, d=0.22 B F_{10}=44.56$, Experiment 3: $M=0.10, S D=0.33, t(250)=5.02, p<$ $.001, d=0.32, B F_{10}>1,000$. An independent samples t-test on the evaluation scores as a function of the targets' typicality condition did not find a significant effect of targets' typicality, Experiment $2: t(574)=1.37, p=.171, d=0.11, B F_{10}=0.23$, Experiment $3: t(513)=1.20, p=.230, d=$ $0.10, B F_{10}=0.20$. In other words, we found no statistically reliable evidence for the effect of group information on the SPF and AMP. In contrast, we found strong evidence for the effect of individuating information on these measures. 


\section{Discussion}

Experiments 2-3 replicated the strong effect of individuating information on self-reported and indirectly measured evaluations. In contrast, the small effect for group information in Experiment 1 did not replicate, as there was no significant effect of group information. Together, these results suggest that group information does not inherently take primacy in perceivers' automatic evaluations. Perceivers strongly relied on the more diagnostic information - their knowledge about personal characteristics of the judged individuals - even in their automatic evaluations.

\section{Additional Experiments and a Meta-Analysis}

The online Supplement Materials report additional experiments that varied various technical details of the stimuli and procedure, mostly to verify that our results are robust to specific methodological choices. These experiments were less informative than the experiments we report in the main text. However, these experiments include conditions that were near replications of Experiment 1. The same is true for the control conditions in Experiments 4-7 (those that tested the effects of potential moderators). Therefore, we meta-analyzed the results from all the relevant conditions in all these experiments, to obtain a more accurate estimation of the effects. The analysis included Experiments 1-3, and only the specific between-participants conditions in Experiments 4-7 and the supplement studies that used the same task instructions and materials as in Experiments 1-3, and that used the same basic paradigm that was used in Experiments 1-3 to present to the participants with both individuating information and group information.

\section{Self-report}

Ten experiments had relevant conditions. Figure 1 shows all the effects and the metaanalytical estimate of the effects. In all these experiments, participants preferred the target individual who behaved positively over the target individual who behaved negatively, with Cohen's $d \mathrm{~s}$ that ranged from 1.48 to 2.09 . A meta-analysis of these experiments, with a random-effects model, found an effect of $d=1.78$ [CI 95\%, 1.66-1.90] that was significantly different from zero, $z$ 
$=28.45, p<.0001$. Cohen's $d$ was calculated using $S_{\text {pooled }}$ (recommended by Lakens, 2013). The calculation of $S_{\text {diff }}$ (recommended by Borenstein et al., 2009) resulted in an identical meta-analytic effect (and similar confidence-intervals).

Individuation

\section{Experiment}

Experiment 1

Experiment 2

Experiment 3

Experiment 4 (control)

Experiment 5 (control)

Experiment 6 (control)

Experiment 7 (control)

Experiment S1

Experiment S11 (control)

Experiment S12 (control)

\section{Group}

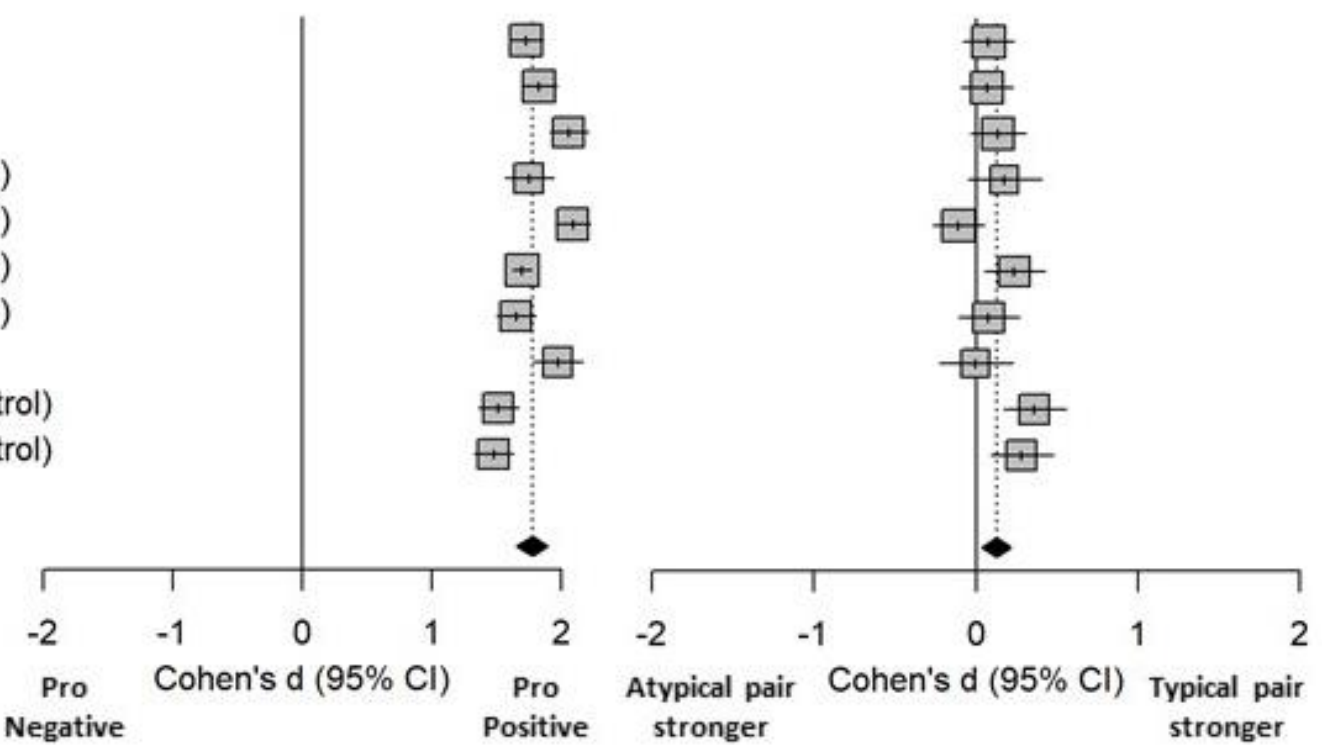

Figure 1. Meta-Analyses results for the effect of individuating information (left panel) and of group information (right panel) on self-reported evaluations. In the left panel, the "Pro-positive" label indicates a preference for the target individual who behaved positively. In the right panel, the "Typical pair stronger" label indicates that the preference for the positively-portrayed target over the negatively-portrayed target was stronger when they were typical of their respective group than atypical of their groups.

The meta-analysis found that the preference between the individuals was slightly affected by the group-membership of the individuals. Within each experiment, we computed Cohen's $d$ for the difference between the preference when the targets were typical of their group and when the targets were atypical of their group. These effects size ranged from -0.11 to 0.36 . As reported in Experiments 1-3, these effects often did not reach statistical significance in each individual 
experiment. However, a meta-analysis with a random-effects model estimated an effect of $d=0.13$ [CI 95\%, 0.04-0.22] that was significantly different from zero, $z=2.95, p=.003$.

Overall, the results of the meta-analysis of the self-report measures confirmed the large effect of individuating information on participants' self-reported evaluations. Yet, the meta-analysis also revealed that group information had a very small, but statistically reliable, effect on the selfreported evaluations of the individual group members.

\section{Indirectly-measured evaluation}

We found conditions relevant to this meta-analysis in 12 of our experiments. Figure 2 shows all the effects and the meta-analytical estimate of the effects. In all these experiments, the results of the indirect measure suggested that participants had an automatic preference for the target individual who behaved positively over the target individual who behaved negatively, with Cohen's $d \mathrm{~s}$ for the preference scores that ranged from 0.20 to 0.71 . A meta-analysis of all these experiments, with a random-effects model, found an effect of $d=0.42$ [CI 95\%, 0.34-0.49] that was significantly different from zero, $z=11.23, p<.0001$.

Group information had a small to moderate effect on the preference between the individuals: Cohen's $d$ s for the difference between the preference between the typical targets and the preference between the atypical targets ranged from 0.00 to 0.66 . A meta-analysis of that effect, with a random-effects model, found an effect of $d=0.23$ [CI 95\%, 0.14-0.33] that was significantly different from zero, $z=4.66, p<.0001$. Similar to the self-reported evaluations analysis, the results of these meta-analyses suggest strong evidence of a sensitivity to individuating information in participants' indirectly-measured evaluations. However, the meta-analysis also revealed a small, yet statistically reliable, effect of the group information.

\section{Estimating the discrepancy between direct and indirect measures}

The estimated effect of group information on indirectly measured evaluation was somewhat larger than the estimated effect of group information on self-reported evaluation, although with some overlap between their confidence intervals. Further, the estimated effect of individuating 
information was much larger on self-reported evaluation than on indirectly measured evaluation. Therefore, the results of the meta-analysis could suggest that although individuating information was the dominant influence on both directly and indirectly measured evaluation, that dominance was more pronounced for directly-measured evaluation than for indirectly measured evaluation.

Individuation

\section{Experiment}

Experiment 1

Experiment 2

Experiment 3

Experiment 4 (control)

Experiment 5 (control)

Experiment 6 (control)

Experiment 7 (control)

Experiment $\mathrm{S} 3$

Experiment $\mathrm{S} 4$

Experiment $\mathrm{S} 5$

Experiment S11 (control)

Experiment $\mathrm{S} 12$ (control)
Group

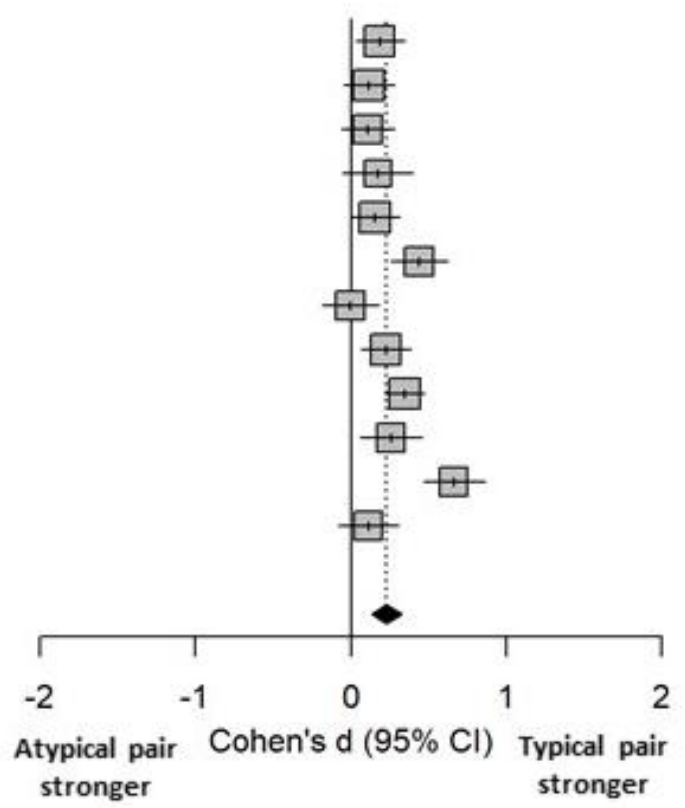

Figure 2. Meta-Analyses results for the effect of individuating information (left panel) and of group information (right panel) on indirectly-measured evaluations. In the left panel, the "Pro-positive" label indicates a preference for the target individual who behaved positively. In the right panel, the "Typical pair stronger" label indicates that the preference for the positively-portrayed target over the negatively-portrayed target was stronger when they were typical of their respective group than atypical of their groups.

To compare the extent of the dominance of individuating information over group information on direct evaluation measures relatively to indirect measures, we compared the sizes of the effects of individuating information and group information, between direct and indirect measures, after adjusting the effect-sizes for each measure's reliability. This procedure verifies that the obtained difference in effect sizes does not stem from low reliability of one measure, relative to 
the other (Baugh, 2002; Gawronski et al., 2021; see Navon et al., 2021 for a similar analysis strategy).

We first computed the adjusted effect-sizes of the individuation and group effects on selfreport and on the indirect measure. Then, for each measure, we computed the difference between the two effect-sizes. This was the estimate of the relative size of the two effects, within each measure. Finally, we computed the difference between these two differences. For each difference score (i.e., the differences between the two effects, and the difference of differences), we computed the $95 \%$ confidence-intervals around that difference using a bootstrapping procedure, with the $\mathrm{R}$ packages rsample (version 0.1.1, Silge et al., 2021) and bayestestR (version 0.11.5, Makowski et al., 2019). Table 3 presents the results of this test for each experiment that contributed to the metaanalysis of both self-reported evaluation and indirectly-measured evaluation. Self-reported evaluation was always much more sensitive to individuating information than to group information. The indirectly measured evaluation was also usually sensitive to individuating information more than to group information. Yet, the dominance of individuating information over group information was always stronger in directly-measured evaluation than indirectly-measured evaluation. These results, together with the results of the meta-analyses reported above, strengthen the interpretation of our findings as suggesting a stronger influence of group information on indirectly measured evaluation than on self-reported evaluation, relatively to the effect of individuating information on these measures. 
Table 3

A comparison of the effects of individuating and group information on direct vs. indirect evaluation measures

Difference

\begin{tabular}{|c|c|c|c|}
\hline \multirow[b]{2}{*}{ Experiment } & \multicolumn{2}{|c|}{ Individuating information vs. Group information } & \multirow[t]{2}{*}{ Indirect vs. Self-report } \\
\hline & Self-report measure & Indirect measure & \\
\hline 1 & $0.90 * * *[0.71 ; 1.93]$ & $0.25 * *[0.06 ; 0.46]$ & $-0.65 * * *[-0.94 ; 0.45]$ \\
\hline 2 & $1.06 * * *[0.88 ; 1.37]$ & $0.16[-0.03 ; 0.59]$ & $-0.90 * * *[-1.20 ;-0.53]$ \\
\hline 3 & $1.17 * * *[0.99 ; 1.53]$ & $0.26 * *[0.09 ; 0.47]$ & $-0.91 * * *[-0.70 ; 0.00]$ \\
\hline 4 & $1.27 * * *[1.10 ; 1.60]$ & $0.50 * * *[0.32 ; 0.81]$ & $-0.77 * * *[-1.09 ;-0.50]$ \\
\hline 5 & $0.79 * * *[0.57 ; 1.09]$ & $0.03[-0.18 ; 0.27]$ & $-0.76 * * *[-1.06 ;-0.52]$ \\
\hline 6 & $0.95 * * *[0.73 ; 1.27]$ & $0.24 *[0.03 ; 0.66]$ & $-0.71 * * *[-1.01 ;-0.29]$ \\
\hline 7 & $0.85 * * *[0.57 ; 1.24]$ & $0.35 *[0.11 ; 0.70]$ & $-0.50 * *[-0.87 ; 0.17]$ \\
\hline S11 & $0.51 * * *[0.29 ; 0.82]$ & $-0.23 *[-0.46 ;-0.03]$ & $-0.74 * * *[-1.04 ;-0.53]$ \\
\hline S12 & $0.57 * * *[0.34 ; 0.87]$ & $0.08[-0.19 ; 0.42]$ & $-0.49 * *[-0.83 ;-0.16]$ \\
\hline
\end{tabular}

Note. The difference within each measure was computed as [effect of individuating - effect of group]. The difference between those differences was computed as [indirect measure - direct measure]. $* p<.05, * * p<.01, * * * p<.001$.

\section{Experiment 4: Moderation by the Amount of Group Information}

In the first part of our research, we established that our basic paradigm shows a large effect of individuating information on the directly and indirectly measured evaluation of individual group members. These results suggest that automatic evaluation of individual group members is not inherently determined by group information. In contrast, previous studies found that when the target individuals belonged to known (rather than fictional) social groups, group information often dominated the automatic judgment of those individuals (Cao \& Banaji, 2016; Navon et al., 2021). Therefore, our next goal in the present research was to test whether manipulating possible features that distinguish between known groups and previously unknown groups would moderate the effect of group information in our paradigm.

One straightforward distinction between the present research and past research is that participants were probably exposed to information about more than four individual members of 
each of the social groups that appeared in the previous research (Black/White people, old/young people, women/men). In other words, one factor that might increase the effect of group information on evaluation of individual members is the amount of information known about the groups. More evidence that the members of a certain group misbehave may increase the certainty that negative behavior characterizes all members of the group. Clearly, a person who knows seven misbehaving group members and one well-behaving group member would be more likely to infer that something is wrong with this group than a person who knows only three misbehaving group members and one well-behaving group member. Therefore, a straightforward means to increase the effect of the evaluation of the group information on the evaluation of individual members of that group would be strengthening the evaluation of the group by exposing the participants to more group members whose characteristic behaviors are of the typical valence of that group.

A further reason for the hypothesized moderating effect of the number of typical group members is that people might be less likely to individualize group members, the more members they know. The information people accrue about members of social groups is often compatible with certain stereotypes and prejudice, not only due to motivational reasons (e.g., Brewer, 1988; Fiske \& Neuberg, 1990; Oakes et al., 1994; Spears et al., 1997; Tajfel et al., 1979), but also because of cognitive limitations that encourage perceivers to focus on the perceived similarities between the group members (e.g., Allport, 1954; Rothbart et al., 1978; Stangor \& Ford, 1992; Stangor \& McMillan, 1992; Von Hippel et al., 1993). What may begin as a complex and detailed representation of individual group members would later transform to a simpler and schematic representation of the whole group that is learned and transmitted with ease (Kirby et al., 2008; Martin et al., 2014). In other words, people might be more likely to rely on the schematic group representation when they perceive and judge specific group members, the more people they know from that group.

On the other hand, the amount of information might not increase the effect of group information on the judgment of atypical group members because it might be relatively easy to 
remember the individuating information of one atypical group member when the other members are clearly of opposite valence. In other words, the atypicality of the atypical group member might be more salient with the increase of the number of typical group members that the perceiver knows. As a result, increasing the number of typical group members might not increase the effect of group information on the judgment of the atypical group member. Rather, it might even further decrease that influence ${ }^{3}$.

In Experiment 4, we investigated whether group information would have a stronger influence on the evaluation of individual group members if we expose participants to a larger number of group members whose behaviors are of the group's typical valence. We compared our basic paradigm (three typical group members in each group) with a modification of that paradigm that exposed participants to seven typical group members. In both conditions, there was only one atypical group member within each group. We examined whether the effect of group information on directly and indirectly measured evaluation of individual members would be larger when the number of typical group members is larger.

\section{Methods}

\section{Participants}

The details about the participants appear in Table 1. We applied the same exclusion criteria as in Experiment 1. To try to save data collection resources, we planned two data collection stops, at the cost of reducing the critical p-value to .029 (computed using paugmented.r; Sagarin et al., 2014). We eventually collected the full sample size $(\mathrm{N}=600)$.

\section{Materials}

\footnotetext{
${ }^{3}$ In experiment S7 in the supplement, we tested whether participants subtyped the atypical group member and did not consider him a member of the group. We tested whether participants would rely on group membership when judging whether a non-evaluative attribute characterizes the atypical group member. We provided information about whether that attribute characterizes the typical group members of the group and no information about whether it characterizes the atypical group member. We found that participants used the group information when judging the atypical group member on the non-evaluative attribute. Thus, it seems that our paradigm does not encourage participants to rob the atypical group member from his group membership (i.e., to subtype the atypical group member).
} 
Face dataset. The faces were the same as in the previous experiments, except for the visual grouping cue: a colored frame instead of a fictitious skin tone (Experiment S5 in the online supplement found that this visual group cue produced the same results as the skin tone cue). We created two versions of the visual grouping cue: a purple frame, and an orange frame. For each participant, we randomly selected eight (or sixteen) face identities as the members of the groups. All the photos of all the members of each group (four or eight face identities) had the same frame color.

\section{Procedure}

The procedure was identical to Experiments 1, except for the number of group members presented in the induction part. In that part, we manipulated between participants the size of the groups. In the large group condition, we presented seven typical members and one atypical member from each group. The control, small group condition presented three typical members and one atypical member from each group, as in the previous experiments. The induction task included 32 trials in the small group condition (as before), and 64 trials in the large group condition. All other characteristics were the same as in Experiments 1.

\section{Results}

\section{Individuation and group effects}

Self-report. Table 4 presents the descriptive statistics and reliability (internal consistency) of each measure. We submitted the self-reported evaluation scores of the two targets to a 2 (valence of target's valence: positive, negative) x 2 (targets' typicality: typical, atypical) x 2 (group size condition: small, large) ANOVA. As expected, target's valence had a large main effect, $F(1,597)=$ 686.04, $p<.001, \eta^{2}{ }_{p}=.53$, reflecting a strong sensitivity to individuating information. There were no other effects in the ANOVA, $p s>.029$. Notably, targets' typicality did not moderate the effect of target's valence, $F(1,597)=0.05, p=.832, \eta^{2}{ }_{p}<.001$, and that (lack of) interaction was not moderated by the amount of group information, $F(1,597)=3.70, p=.055, \eta^{2}{ }_{p}=.006$. Thus, the 
experiment found no statistically reliable evidence that group information influenced self-reported evaluations, regardless of group size.

IAT. Overall, IAT scores reflected high sensitivity to the individuating information, with a preference for the positively portrayed target over the negatively portrayed target, $M=0.21, S D=$ $0.42, t(600)=12.57, p<.001, d=0.51, B F_{10}>1,000$. There was a small effect of the targets' typicality that did not reach our corrected critical p-value of $.029, F(1,597)=4.09, p=.044, \eta^{2}{ }_{p}=$ .007. Descriptively, that reflected a stronger pro-positive preference for targets that were typical to their group $(M=0.25, S D=0.40)$ than for atypical targets $(M=0.18, S D=0.44)$. That nonsignificant effect was not moderated by the amount of group information, $F(1,597)=0.01, p=$ $.908, \eta^{2}{ }_{p}<.001$, providing no evidence that group size moderated the effect (or the lack of it) of group information on automatic evaluation.

Table 4

Experiment 4: Descriptive statistics for the preferences between the group members

\begin{tabular}{lllllll}
\hline Group size & Targets' typicality & Preference & $N$ & Mean & SD & $\alpha^{\text {a }}$ \\
\hline Small & Typical pair & Self-report & 145 & 3.54 & 2.56 & $0.79,0.87$ \\
& & IAT & 145 & 0.26 & 0.42 & 0.82 \\
& Atypical pair & Self-report & 152 & 2.99 & 3.65 & $0.82,0.92$ \\
& & IAT & 152 & 0.19 & 0.44 & 0.81 \\
Large & Typical pair & Self-report & 148 & 3.34 & 2.99 & $0.81,0.82$ \\
& & IAT & 148 & 0.24 & 0.40 & 0.81 \\
& Atypical pair & Self-report & 156 & 3.79 & 3.44 & $0.85,0.93$ \\
& & IAT & 156 & 0.18 & 0.44 & 0.80 \\
\hline
\end{tabular}

Note. In all measures, positive values reflect a preference for the positively portrayed individual over the negatively portrayed individual. ${ }^{a}$ For the self-reported evaluations, the left value refers to the Positive member items, and the right value refers to the Negative member items.

\section{Discussion}

Experiment 4 tested whether the influence of group membership on the evaluation of individual group members would increase when the groups are comprised of seven typical members 
and one atypical member, compared with three typical members and one atypical member. However, no differences emerged between these two conditions. Had we found a difference between these conditions, that would have provided initial support for the hypothesis that people are more likely to rely on group information when the information is more consistent and more abundant. The lack of moderation by this factor further generalizes our finding that automatic evaluation of individual group members is only slightly sensitive to group information. It decreases the likelihood that the difference between our results and previous results with pre-existing social group (Cao \& Banaji, 2016; Navon et al., 2021; Rubinstein et al., 2018) is due to a larger amount of information that people have on pre-existing than on novel social groups.

\section{Experiment 5: Moderation by Group Salience Upon Judgment}

In Experiments 5, we tested the moderating effect of the salience of the target individual's group membership, upon judgment. Individual group members from existing social groups (e.g., race, gender) are categorized quickly (as fast as $100 \mathrm{~ms}$ ) upon exposure to visual characteristics of the group that appear in the individual's face (Ito \& Urland, 2003, 2005; Quinn et al., 2010). Following that categorization, group information is often activated automatically, and influences judgment (Blair, 2002; Blair \& Banaji, 1996; Bodenhausen \& Macrae, 1998; Fazio et al., 1995; Kawakami \& Dovidio, 2001; Kunda et al., 2002; Kunda \& Spencer, 2003; Macrae et al., 1995; Stangor et al., 1992). Group information could have a weaker influence on judgment when the group is less salient upon judgment (e.g., Brewer, 1979; Mullen et al., 1992). For example, the automatic preference (inferred from IAT scores) for an individual member of a dominant group (White people) over an individual member of a stigmatized group (Black people) was smaller when the stimuli that represented the individuals in the IAT were their names rather than their photos. Deliberate, self-reported evaluation was not sensitive to this manipulation (Navon et al., 2021).

To test the moderating effect of group salience on the influence of group information on the evaluation of individual group members, we created three versions of the evaluation measures, varying the salience of the group membership of the target individuals from low, to medium (as in 
Experiments 1-3) to high. In a pre-test of the high salience condition in Experiment S2 (online Supplementary Materials), the effect of group information on automatic evaluation was stronger than the estimated effect in the meta-analysis of the effect in the basic paradigm. However, comparing between different experiments is problematic, if only because of the passage of time, which might lead to differences in the samples that participated in each experiment. Therefore, in Experiment 5, we compared directly the relatively low salience of the group in our previous experiments and a condition of relatively high salience of the group membership (as in Experiment S2). We also added a medium salience condition.

\section{Method}

\section{Participants}

The details about the participants appear in Table 1. We applied the same exclusion criteria as in Experiment 1. We planned to collect data from at least 1,200 participants to achieve reasonable power for our effects of interest. We eventually collected data from 1,984 participants because technical reasons prevented us from ending the data collection on time.

\section{Materials}

Face dataset. As in Experiment 4, we used a colored frame as the visual cue of group membership. It was easier to manipulate the presence of that visual cue than the skin tone cue used in Experiments 1-3.

\section{Procedure}

The procedure was identical to Experiments 1, except for the materials used in the evaluation measures, and for the removal of a group evaluation measures from the end of the experiment, to save time. In the evaluation measures, we manipulated between participants the salience of the group membership of the individual targets' social groups. All other characteristics were the same as in Experiments 1.

IAT. In the Low group salience condition, the target individuals' IAT-category labels and the specific items of each category were the faces of each individual, without the visual group cue 
(i.e., the colored frame). In the Medium group salience condition, the category labels and items were the faces of each individual with the visual cue. In the High salience condition, the category labels were textual - referring to the group membership of the target (e.g., for a member of the Baunifs, the label was The Baunif), and the items of each category were the faces of each target individual, with the visual cue of the group (see Figure 3).

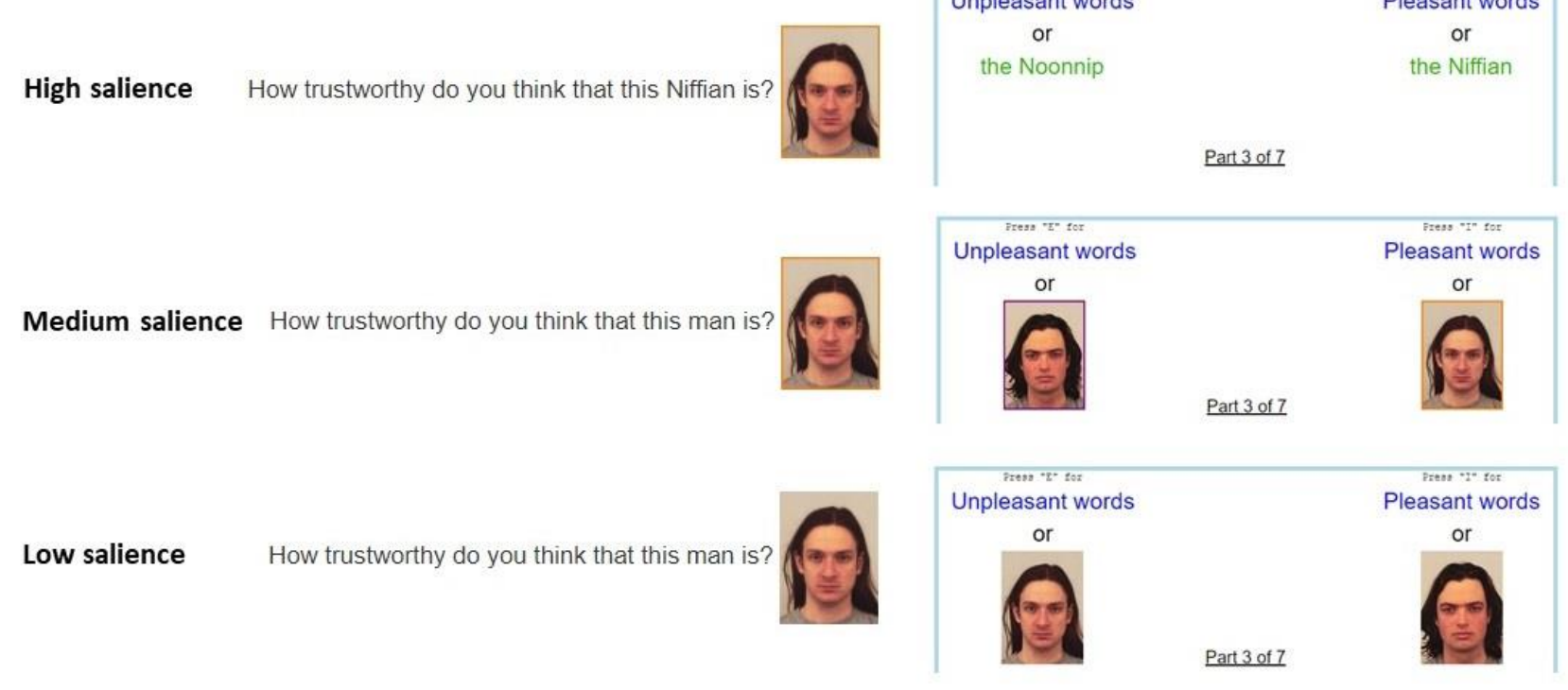

Figure 3. Examples for self-report items (left) and IAT category labels (right) in the Low, Medium, and High salience conditions. Notice that in the Low salience condition, the photo of the target person does not include a colored frame (the visual cue for the group).

Self-reported evaluation. As in the previous experiments, each question referred to one target individual and showed the individual's face below the question. We manipulated the language that referred to each individual and the inclusion of the groups' visual cue in the photo. In the Low group salience condition, the questions' text referred to the target individuals as this man and the face of each individual was presented without the visual cue of the group. In the Medium salience condition, the questions' text referred to the target individual as this man, but the face of that individual was presented with the visual cue. Finally, in the High salience condition, the questions' text referred to the individuals by the group name (e.g., for a member of the Baunifs, the 
individual was called The Baunif). In that condition, as in the medium salience condition, the face of the target individual appeared with the visual cue of the group (Figure 3).

\section{Results}

\section{Individuation and group effects}

Self-report. Table 5 presents the descriptive statistics and reliability (internal consistency) of each measure. We submitted the self-reported evaluation score to a 2 (valence of target's valence: positive, negative) x 2 (targets' typicality: typical, atypical) x 3 (salience condition: low, medium, high) ANOVA. A main effect of target's valence, $F(1,1876)=3,200.76, p<.001, \eta^{2}{ }_{p}=$ .63 , reflected a strong sensitivity to individuating information. There were no other significant effects in the ANOVA, $\eta^{2}{ }_{p}<.004, p s>.060$. Two absent effects were of most interest. First, there was no moderation of the effect of individuating information by the typicality of the targets to their group, $F(1,1876)=1.04, p=.308, \eta_{p}^{2}<.001$, compatible with the possibility that group information did not influence self-reported evaluation. Second, this group effect (or lack there-of) was not moderated by the salience condition, $F(1,1876)=1.90, p=.150, \eta^{2} p=.002$, compatible with the possibility that a high salience of the group upon judgment did not increase the effect of the group information on self-reported evaluation.

IAT. On average, the IAT scores reflected high sensitivity to the individuating information, with a preference for the positively portrayed target over the negatively portrayed target, $M=0.30$, $S D=0.43, t(1881)=27.90, p<.001, d=0.64, B F_{10}>1,000$. In a 2 (targets' typicality) $\times 3$ (salience condition) ANOVA on the IAT scores, we found a small effect of the targets' typicality, $F(1,1876)$ $=15.56, p<.001, \eta_{p}^{2}=.008$, reflecting a stronger preference for the positively portrayed target when the targets' valence was compatible with most members of their group $(M=0.32, S D=0.33)$ than when their valence was opposite $(M=0.24, S D=0.28)$. Importantly, that effect was moderated by the salience condition, $F(1,1876)=15.18, p<.001, \eta^{2}{ }_{p}=.02$. As Table 4 and Figure 4 show, the pattern of moderation reflected a stronger moderation by the targets' typicality (i.e., sensitivity to the group information) when the salience was higher rather than lower. Holm-Bonferroni corrected 
post-hoc tests ${ }^{4}$ found an effect of group information under high salience, $t(1,876)=6.30, p<.001, d$ $=0.44, B F_{10}>1,000$, but not under low salience, $t(1,876)=-1.39, p=.166, d=-0.12, B F_{10}=0.165$, or medium salience, $t(1,876)=1.80, p=.107, d=0.15, B F_{10}=0.310$. Note that even in the highsalience condition, when target individuals were atypical of their group, the IAT still showed a preference for positively-portrayed individual over the negatively-portrayed individual, $t(308)=$ $5.27, p<.001, d=0.30$.

Table 5

Experiment 5: Descriptive statistics for the preferences between the group members

\begin{tabular}{lllllll}
\hline Group salience & Targets' typicality & Preference & $N$ & Mean & $S D$ & $\alpha^{\text {a }}$ \\
\hline Low & Typical pair & Self-report & 323 & 3.52 & 2.69 & $0.83,0.88$ \\
& & IAT & 323 & 0.26 & 0.36 & 0.73 \\
& Atypical pair & Self-report & 326 & 3.85 & 2.91 & $0.80,0.87$ \\
& & IAT & 326 & 0.30 & 0.41 & 0.79 \\
\multirow{3}{*}{ Medium } & Typical pair & Self-report & 297 & 3.44 & 2.81 & $0.77,0.86$ \\
& \multirow{3}{*}{ Atypical pair } & IAT & 297 & 0.32 & 0.40 & 0.81 \\
& & Self-report & 325 & 3.75 & 2.92 & $0.84,0.87$ \\
& Typical pair & IAT & 325 & 0.26 & 0.41 & 0.80 \\
& & Self-report & 302 & 4.03 & 2.54 & $0.73,0.86$ \\
& Atypical pair & IAT & 302 & 0.38 & 0.46 & 0.85 \\
& & Self-report & 309 & 3.80 & 3.22 & $0.84,0.88$ \\
& & IAT & 309 & 0.16 & 0.52 & 0.88 \\
\hline
\end{tabular}

Note. In all measures, positive values reflect a preference for the positively portrayed individual over the negatively portrayed individual. ${ }^{\text {a }}$ For the self-reported evaluations, the left value refers to the Positive member items, and the right value refers to the Negative member items.

\footnotetext{
${ }^{4}$ Corrected for three comparisons. Bayes-factors for post-hoc tests were corrected for multiple comparisons using the Westfall approach (Westfall et al., 1997).
} 


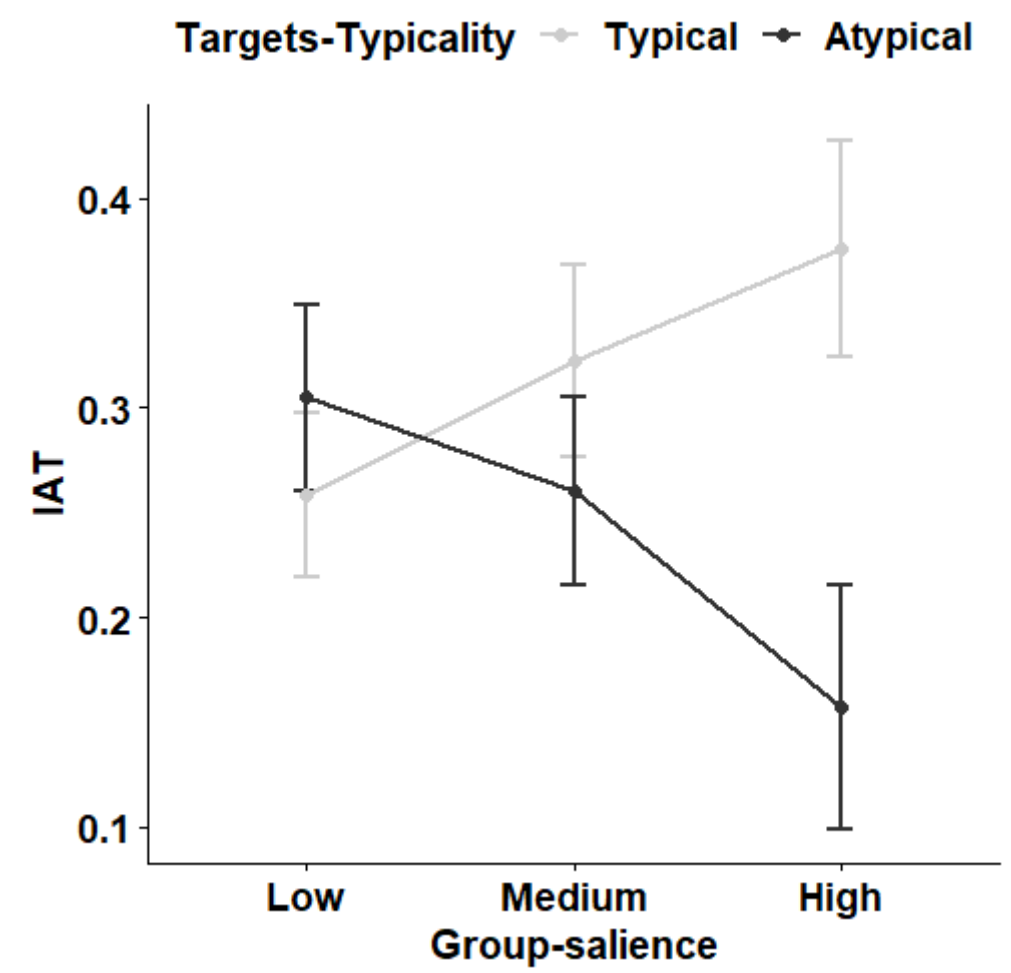

Figure 4. IAT scores as a function of the group salience and targets-typicality conditions in Experiment 5. Error-bars indicate the 95\%-confidence interval.

\section{Discussion}

In Experiment 5, we found that IAT scores, but not self-report, were sensitive to the extent to which the group membership was salient upon judgment. In their self-reports, participants preferred the target individual that behaved positively irrespective of his group valence, and that preference did not decrease even when his membership in the group was highly salient upon judgment. In contrast, although IAT scores always reflected a preference for the target individual that behaved positively, group information influenced that preference when the group membership was highly apparent throughout the judgment task. This is compatible with previous findings that group salience increases the effect of group information on the automatic, but not the deliberate, judgment of individual group members (Navon et al., 2021).

\section{Experiments 6-7: Moderation by Motivational Relevance}

In Experiment 6 and 7, we tested another factor that is often relevant for existing social groups and might increase the influence of group information on evaluation - the membership of the perceiver in one of the social groups. People's ingroup is generally more motivationally 
relevant, compared with outgroups (e.g., Brewer, 1988; Van Bavel et al., 2011), leading often to biases in favor of ingroup members over the outgroup. For example, people devote more processing resources to faces of ingroup members than outgroup members (e.g., Bernstein et al., 2007), attribute more positive traits and qualities to their ingroup relative to outgroups (e.g., AshburnNardo et al., 2001; Brewer \& Silver, 1978; Ferguson \& Kelley, 1964; Ratner et al., 2014), and distribute more resources to their ingroup over an outgroup (Brewer \& Silver, 1978; Oakes \& Turner, 1980; Tajfel, 1970).

Moreover, and especially relevant to the present research, theory regarding automatic judgment has suggested that scores on indirect measures could reflect the operation of two opposing judgments - a judgment toward the respondent's ingroup, and a judgment toward the socially dominant group (Axt et al., 2014; Dasgupta, 2004; Stern \& Axt, 2019). Support for this theoretical claim comes from ample research showing that people's automatic judgments of social groups are heavily moderated by their membership in these groups (i.e., known-groups validity in automatic judgment measurement, e.g., Banse et al., 2001; Charlesworth \& Banaji, 2019; De Houwer, 2006; Greenwald et al., 1998). Together, these theories and research suggest that the judgment of individual group members would be moderated by the participants' membership in one of the social groups. We tested that moderating effect, and the strength of its influence, on deliberate and automatic judgments of individuals.

In Experiment 6 and 7, we told half of the participants that they belong to one of the groups in our basic paradigm. That assignment might motivate the participants to judge ingroup members more favorably than outgroup members, to improve their own self-evaluation. Existing social groups such as race, age, and gender, often include the perceiver who is making the judgment (e.g., a White perceiver who is judging White and Black individuals). For that reason, in research on the judgment of individual group members of existing social groups, it is hard to disentangle the effect of ingroup bias from the effect of prejudice and stereotypes (i.e., the effect of group schemas on judgment). The use of novel social groups provides a valuable tool for investigating this issue 
because it allows manipulating, separately, the information about the groups, the information about the individuals, and whether there is an intergroup context that involves the perceiver. Experiments 6-7 tested whether perceivers' membership in one of the judged groups would increase the perceivers' reliance on group information in the judgment of individual group members.

We first piloted the effect of assigning the participant to one of the two novel groups (a minimal group manipulation, as in Pinter \& Greenwald, 2011; see also Dunham, 2013) in Experiment S12 (reported in the online supplement). In that experiment, all the participants were told that they belong to one of the two novel groups. We found a stronger effect of the group membership information on the IAT $(d=0.88)$ than in Experiment $1(d=0.18)$, and no effect of group information on self-reported evaluation (comparable to what we found in the previous experiments). These results might suggest that group information has a larger influence on automatic, but not on deliberate evaluation, when the participant perceives the social groups as an ingroup and an outgroup. To test that possibility experimentally, in Experiments 6 and 7, we assigned half of the participants to one of the target groups, using a minimal-group manipulation. The other half were control participants and completed a study similar to Experiment 1 . We tested whether the minimal-group manipulation would increase the effect of group information on automatic evaluation, using the IAT in Experiment 6 and the Evaluative Priming Task (EPT; Fazio et al., 1986, 1995) in Experiment 7. We also tested whether that manipulation would have no influence on deliberate evaluation, as in Experiment S12, and our results with the previous moderator (group salience). Further, the control condition in Experiment 7 extended our conceptual replications of Experiments 1-3 with a fourth supposed measure of automatic evaluation (the EPT). In the minimal group procedure, participants rehearsed the information that one of the two groups is their group, and the other group is not. To increase the likelihood that the participant would identify with his/her minimal ingroup, the valence of the participant's group was always positive, and the valence of the outgroup was always negative. That decision did not harm our ability to test whether group membership has a stronger influence when the perceiver belongs to 
one of the groups. If, when participants judge the atypical group members, their preference for the positively portrayed individual over the negatively portrayed individual would be smaller when the negatively portrayed individual is a member of their ingroup than when the participant is not assigned to any of these groups, that would suggest that group information had a larger effect on judgment when the perceiver is a member of one of the groups than when the perceiver is not personally involved with any of the groups.

\section{Method}

\section{Participants}

The details about the participants appear in Table 1. As in Experiment 1, in Experiment 7 we excluded participants who had $>10 \%$ fast trials in the IAT (RT<300 ms; see Greenwald et al., 2003). In Experiment 8 we excluded participants with > 40\% error trials in the EPT (Bar-Anan \& Nosek, 2014). In the preregistration of the two experiments, we stated that we would recruit female and male participants separately, with each sub-sample $(\mathrm{Ns}=1,000)$ reaching the required statistical power (at least $80 \%$ ). That statement was a mistake, as we did not intend to make any comparison between participants' genders (see preregistration file on OSF). Thus, we eventually collected data from a total of 1,000 participants on each experiment, rather than 2,000, collapsing the data across genders.

\section{Materials}

The group names and behavior pool were the same as in Experiments 1-3. The IAT and selfreport were the same as in Experiment 1.

Faces dataset. To increase the likelihood of a successful minimal group manipulation, we matched the gender of the targets to the gender of the participant. That also allowed generalizing our results to female targets. The male faces were the same faces used in the previous experiments. Like the males faces, the female faces ( 24 face identities, as the male faces) were taken from the KDEF dataset (see all the face in the online supplement). We used a colored frame as the visual cue of the categories (as in Experiments 4 and 5). 


\section{Procedure}

Half of the participants, those randomly assigned to the Minimal Group Paradigm (MGP) condition, started the experiment with a minimal-group induction task. The rest of the tasks were the same in both conditions, and identical to Experiment 1: the behavioral information task followed by the evaluation measures. At the end, participants completed a group-identification measure, as a manipulation-check. On Experiment 6, the measure of automatic evaluation was the IAT (identical to Experiment 1), and on Experiment 7 it was the EPT. All other characteristics were the same across the two experiments. The online Supplementary Materials include a detailed description of all task instructions.

Minimal group induction. Only participants in the MGP condition completed this part. The minimal-group induction was adapted from Pinter and Greenwald (2011; see also Dunham, 2013). The ingroup consisted of four members (identified by face) and of the participant (although we did not present the participants' photos as stimuli). The outgroup consisted of five members. To increase the similarity between the participant and their group members, all the 9 individuals had the gender self-reported by the participant.

Introduction with the groups. Participants read that they will learn about two groups during the study. They read the groups' names (e.g., the Baunifs and the Lapians), and the visual cue of each group (the color of photo's frame). Participants were then told that they belong to one of these groups (e.g., In this study, YOU BELONG TO THE BAUNIFS GROUP). Participants were always assigned to the positive group, to encourage group identification.

Then, the instructions informed the participants that they would view all members of their ingroup and they were reminded about the visual cue of each group. Participants completed four blocks: The first block consisted of a single trial that presented four faces that appeared together at the center of the screen. Then, on the second and third blocks, they viewed each face separately in the same presentation order (a single trial for each group member on each block), randomized once for each participant. Each face appeared for five seconds, and the intertrial interval was $750 \mathrm{~ms}$. 
Finally, in the last block, participants viewed all faces together again. Across all blocks, a colored frame (either purple or orange, depending on the assigned cue) surrounded the participants' screen, to remind the participants of their group's visual cue.

Categorization task. Following the introduction with the ingroup members, participants practiced the categorization of the group members to the ingroup and outgroup. The task consisted of two blocks. On each block, participants viewed one stimulus at a time, presented randomly at the center of the screen. Each block consisted of 33 trials: three trials for each stimulus (i.e., 4 ingroup faces, 5 outgroup faces, and 2 group names). The participants' task was to categorize the stimuli as either My Group or Other Group. On the first of these two blocks, a colored frame surrounded the participant's screen, to mark the membership of each face. Each group-name stimulus appeared with a colored frame and printed in the same color (the group color). The second block was identical to the first one, but, there were no visual cues (colored frame of font). This encouraged participants to learn the identity of each face, and not just the visual cue. On both blocks, participants received feedback for their response (i.e., correct / wrong).

Evaluative Priming Task (EPT). The EPT (Fazio et al., 1986, 1995) is a sequential priming task that has been widely used as a measure of automatic evaluation (Cameron et al., 2012). The primes in the task were the faces of the target pair (the five photos used in the IAT for each target). Participants classified twenty target words to the categories Pleasant words (items: positive, pleasant, good, outstanding, beautiful, magnificent, marvelous, excellent, appealing, delightful) and Unpleasant words (negative, bad, horrible, miserable, hideous, dreadful, painful, repulsive, awful, ugly). The task consisted of three blocks, each containing 60 trials (15 trials per prime-target combination). The trial sequence was based on a previous set of studies that showed good measurement quality (Navon et al., 2021). Each trial started with a fixation stimulus displayed for $500 \mathrm{~ms}$, replaced by a prime stimulus that appeared for $200 \mathrm{~ms}$, until the appearance of the target words that appeared until the participant's response. After error responses, a red X appeared for $1500 \mathrm{~ms}$. The inter-trial interval was $500 \mathrm{~ms}$. Before the score computation, all latencies were log- 
transformed, and outlier trials and participants were excluded. We excluded incorrect responses and trials with latency exceeding the mean of the relevant prime-target condition in 2.5 SDs (Bar-Anan \& Nosek, 2014). Then, for each prime-target condition, within each block, we computed the mean of the log transformed RTs. We subtracted the means of the congruent conditions: Positive targetPleasant and Negative target-Unpleasant from the means of the opposite, incongruent conditions, and divided that difference by the block's standard deviation, similar to the standardization that is performed in the computation of the IAT D score. This standardization was found to outperform other common computations of the scores (e.g., Axt et al., 2020; Bar-Anan \& Nosek, 2014).

Identification with the groups. At the end of the experiment, participants in both conditions completed a questionnaire that assessed the degree to which they identified with each of the two groups, as a manipulation-check to the minimal group manipulation. Participants reported their level of identification with each of the two groups, by rating their agreement on a scale of 1-7 $(1=$ strongly disagree to 7 = strongly agree $)$ with two statements: (a) I feel attached to the [group name] group, and (b) I identify with the [group name] group. The statements appeared in a random order for each participant. We computed a score by averaging the responses to the two questions for each group, and then subtracting the average identification with the negative group from the average identification with the positive group. Higher scores indicated stronger identification with the positive group (the ingroup in the MGP condition).

\section{Results}

\section{Group identification (manipulation check)}

Table 6 presents the descriptive statistics and reliability (internal consistency) of each measure.

We submitted the group identification scores to a 2 (group-relevance: MGP, none) $\times 2$ (group: ingroup/positive, outgroup/negative) mixed ANOVA. To test whether participants in the MGP condition identified with their ingroup more strongly than with their outgroup, we focused on the simple effect of group for participants in the MGP condition. On both experiments, participants 
in the MGP condition identified with their ingroup to a greater extent, relative to their identification with the outgroup, $t s(932,921)=22.20,23.09, p s<.001, d s=1.06,1.11$. Participants in the control conditions also showed a stronger identification with the positively portrayed group than with negatively portrayed group, $t s(932,921)=11.96,15.56, p s<.001, d s=0.54,0.70$. We tested the difference between the group identification of the MGP and control conditions by focusing on the interaction effect. That effect reflected smaller identification with the positive group than with the negative group in the control condition relative to the MGP condition, $F(1,932 ; 1,921)=45.48$, $22.25, p s<.001, \eta^{2}{ }_{p}=.05, .02$. The interactions effects were not large, providing evidence only for a rather small effect of MGP on group identification.

Table 6

Experiments 6-7: Descriptive statistics for the preferences between the group members

\begin{tabular}{|c|c|c|c|c|c|c|c|}
\hline Experiment & Relevance & Targets' typicality $^{\text {a }}$ & Measure/Preference & $N$ & Mean & $S D$ & $\alpha^{b}$ \\
\hline \multirow[t]{12}{*}{6} & \multirow[t]{6}{*}{ MGP } & \multirow[t]{3}{*}{ Typical pair } & Self-report & 237 & 4.07 & 2.30 & $0.60,0.86$ \\
\hline & & & IAT & 237 & 0.40 & 0.38 & 0.84 \\
\hline & & & Group identification & 237 & 2.58 & 2.08 & $0.79,0.73$ \\
\hline & & \multirow[t]{3}{*}{ Atypical pair } & Self-report & 249 & 3.76 & 3.05 & $0.88,0.83$ \\
\hline & & & IAT & 249 & 0.01 & 0.45 & 0.88 \\
\hline & & & Group identification & 249 & 1.98 & 2.15 & $0.77,0.85$ \\
\hline & \multirow[t]{6}{*}{ None } & \multirow[t]{3}{*}{ Typical pair } & Self-report & 218 & 3.47 & 2.63 & $0.78,0.88$ \\
\hline & & & IAT & 218 & 0.30 & 0.40 & 0.86 \\
\hline & & & Group identification & 218 & 1.52 & 2.15 & $0.60,0.82$ \\
\hline & & \multirow[t]{3}{*}{ Atypical pair } & Self-report & 230 & 2.76 & 3.33 & $0.88,0.90$ \\
\hline & & & IAT & 230 & 0.11 & 0.46 & 0.89 \\
\hline & & & Group identification & 230 & 1.05 & 2.55 & $0.84,0.86$ \\
\hline \multirow[t]{9}{*}{7} & \multirow[t]{6}{*}{ MGP } & \multirow[t]{3}{*}{ Typical pair } & Self-report & 240 & 3.86 & 2.45 & $0.78,0.87$ \\
\hline & & & EPT & 240 & 0.20 & 0.41 & 0.42 \\
\hline & & & Group identification & 240 & 2.33 & 2.01 & $0.80,0.74$ \\
\hline & & \multirow[t]{3}{*}{ Atypical pair } & Self-report & 242 & 4.01 & 2.87 & $0.89,0.86$ \\
\hline & & & EPT & 242 & 0.05 & 0.39 & 0.48 \\
\hline & & & Group identification & 242 & 2.30 & 2.17 & $0.84,0.82$ \\
\hline & \multirow[t]{3}{*}{ None } & \multirow[t]{3}{*}{ Typical pair } & Self-report & 223 & 3.05 & 2.41 & $0.82,0.82$ \\
\hline & & & EPT & 223 & 0.09 & 0.38 & 0.44 \\
\hline & & & Group identification & 223 & 1.63 & 2.19 & $0.84,0.84$ \\
\hline
\end{tabular}


Table 6

Experiments 6-7: Descriptive statistics for the preferences between the group members

\begin{tabular}{|c|c|c|c|c|c|c|}
\hline Experiment Relevance & Targets' typicality $^{\mathrm{a}}$ & Measure/Preference & $N$ & Mean & $S D$ & $\alpha^{\mathrm{b}}$ \\
\hline & Atypical pair & Self-report & 218 & 2.83 & 3.25 & $0.90,0.90$ \\
\hline & & EPT & 218 & 0.10 & 0.41 & 0.52 \\
\hline & & Group identification & 218 & 1.63 & 2.44 & $0.75,0.83$ \\
\hline
\end{tabular}

Note. In all evaluation measures, positive values reflect a preference for the positive group member over the negative group member. In the group identification measure, positive values indicate stronger identification with the ingroup/the positive group, compared with the outgroup/ the negative group. ${ }^{\text {a }}$ In the MGP condition, the characteristic behaviors of the target from the participant's group were always positive typical pair condition and negative in the atypical pair condition. ${ }^{\mathrm{b}}$ For the self-report and group identification, the left value refers to the alpha for the Positive member (group) items, and the right value refers to the alpha for the Negative member (group) items.

\section{Evaluation}

Self-reported evaluation. We submitted the self-report scores to a 2 (targets' valence: positive, negative) $\times 2$ (targets' typicality of their group: typical, atypical) $\times 2$ (group-relevance: MGP, none) mixed ANOVA. In both experiments, we found a main effect of targets' valence, $F(1,928 ; 1,918)=1,408.20,1,426.70, p s<.001, \eta_{p s}^{2}=.60, .61$, which was slightly moderated by the targets' typicality in Experiment $6, F(1,928)=7.58, p=.006, \eta^{2}{ }_{p}=.008$, but not in Experiment $7, F(1,918)=0.05, p=.829, \eta_{p}^{2}<.001$. The moderation in Experiment 6 reflected a larger propositive preference for typical targets $\left(M_{\text {diff }}=3.78, S D_{\text {diff }}=2.48\right), t(928)=28.09, p<.001, d=1.53$, $B F_{10}>1,000$, than for atypical targets $\left(M_{\text {diff }}=3.28, S D_{\text {diff }}=3.22\right), t(928)=24.94, p<.001, d=$ $1.02, B F_{10}>1,000$ (Holm-Bonferroni corrected post-hoc tests). The moderation by targets' typicality (or lack-there-of) reflects the effect of group information. Importantly, we did not find evidence that the size of that moderation depended on group-relevance, as reflected in a nonsignificant, three-way interaction, $F \mathrm{~s}(1,928 ; 1,918)=1.27,1.02, p \mathrm{~s}=.259, .313, \eta_{p}^{2} \mathrm{~s}=.001$. 
Unexpectedly, in both experiments, the effect of individuating information (i.e., the targets' valence effect) was moderated by the group's relevance, $F(1,928 ; 1,918)=17.56,30.09$, $p s<.001$, $\eta^{2}=.019, .032$. Holm-Bonferroni corrected post-hoc tests showed that the moderation effect reflected stronger (i.e., more extreme) preference for the positive target over the negative target when the participants were assigned to the negative target's group (MGP condition), $t \mathrm{~s}(928,918)=$ $30.12,31.27, p \mathrm{~s}<.001, d \mathrm{~s}=1.44,1.48, B F_{10 \mathrm{~s}}>1,000$, than when they were unrelated to the groups (the control condition), $t \mathrm{~s}(928,918)=23.10,22.35, p \mathrm{~s}<.001, d \mathrm{~s}=1.03,1.03, B F_{10 \mathrm{~s}}>1,000 . \mathrm{We}$ speculate that the MGP induction increased participants' attention to (or interest in) the evaluative information, which led to a stronger effect of the evaluative relevant information (the behaviors of the judged individuals) on the deliberate evaluation of the individuals.

Indirectly-measured evaluation (IAT, EPT). As detailed in Table 4, the preference scores in all the conditions in both experiments were always positive, suggesting an automatic preference for the positively-portrayed target over the negatively-portrayed targets, which reflects the effect of the individuating information. However, the effect of the individuating information was clearly moderated by the manipulations because the preferences were almost zero in some conditions, and much larger in other conditions. We tested these moderations with a 2 (targets' typicality of their group: typical, atypical $) \times 2$ (group-relevance: MGP, none) mixed ANOVA on the preference scores (for the positively-portrayed target over the negatively-portrayed target) in the IAT and EPT.

The ANOVA found no main effect of the group's relevance, $F \mathrm{~s}(1,930 ; 1,919)=0.00,1.57$, $p s=.979,21, \eta_{p s}^{2}<.002$, suggesting no evidence of moderation of the effect of the individuating information by the relevance of the group to the participant. A main effect of target typicality, $F \mathrm{~s}(1$, $930 ; 1,919)=110.33,7.71, p s<.001, .006, \eta_{p s}^{2}=.11, .01$, indicated a moderation of the individuating effect by the group information. In other words, the results suggested that group information influenced the automatic judgment of the individuals. Specifically, the preference scores were much larger when the targets were typical of their groups $(M s=0.35,0.15, S D s=0.39$, $0.40), t s(454,462)=19.27,8.05, p s<.001, d s=0.90,0,37, B F_{10 s}>1,000$, than when they were 
atypical of their groups $(M s=0.06,0.07 S D s=0.45,0.40), t(478,459)=2.75,3.95 . p s=.006,<$ $.001, d s=0.12,0.18, B F_{10 \mathrm{~s}}=2.11,108.45$

The effect of targets' typicality (i.e., group information) was moderated by the group's relevance, $F \mathrm{~s}(1,930 ; 1,919)=13.42,8.17, p<.001, .004, \eta^{2}{ }^{2}=.014, .010$, reflecting a stronger effect of typicality when the participants belonged to one of the groups (the MGP condition), $t \mathrm{~s}(930,919)=10.23,4.08, p \mathrm{~s}<.001, d \mathrm{~s}=0.95,0.37, B F_{10 \mathrm{~s}}>1,000,287.94$, than when they did not belong to any of the groups (the control condition), $t \mathrm{~s}(930,919)=4.74,0.06, p<.001,=1, d=0.44$, $0.00, B F_{10 \mathrm{~S}}>1,000,0.10$ (see Figure 5). In fact, in the MGP condition, when the negativelyportrayed individual was a member of the ingroup and the positively-portrayed individual was a member of the outgroup, there was hardly any evidence for an automatic preference for the positively-portrayed individual $(M s=0.01,0.05, S D s=0.45,0.39), t(248,241)=0.29,2.16, p=$ $0.772, .031, d=0.02,0.14, B F_{10}=0.073,0.709$, suggesting a near equal effect of the individuating information and the group membership on automatic evaluation.

A
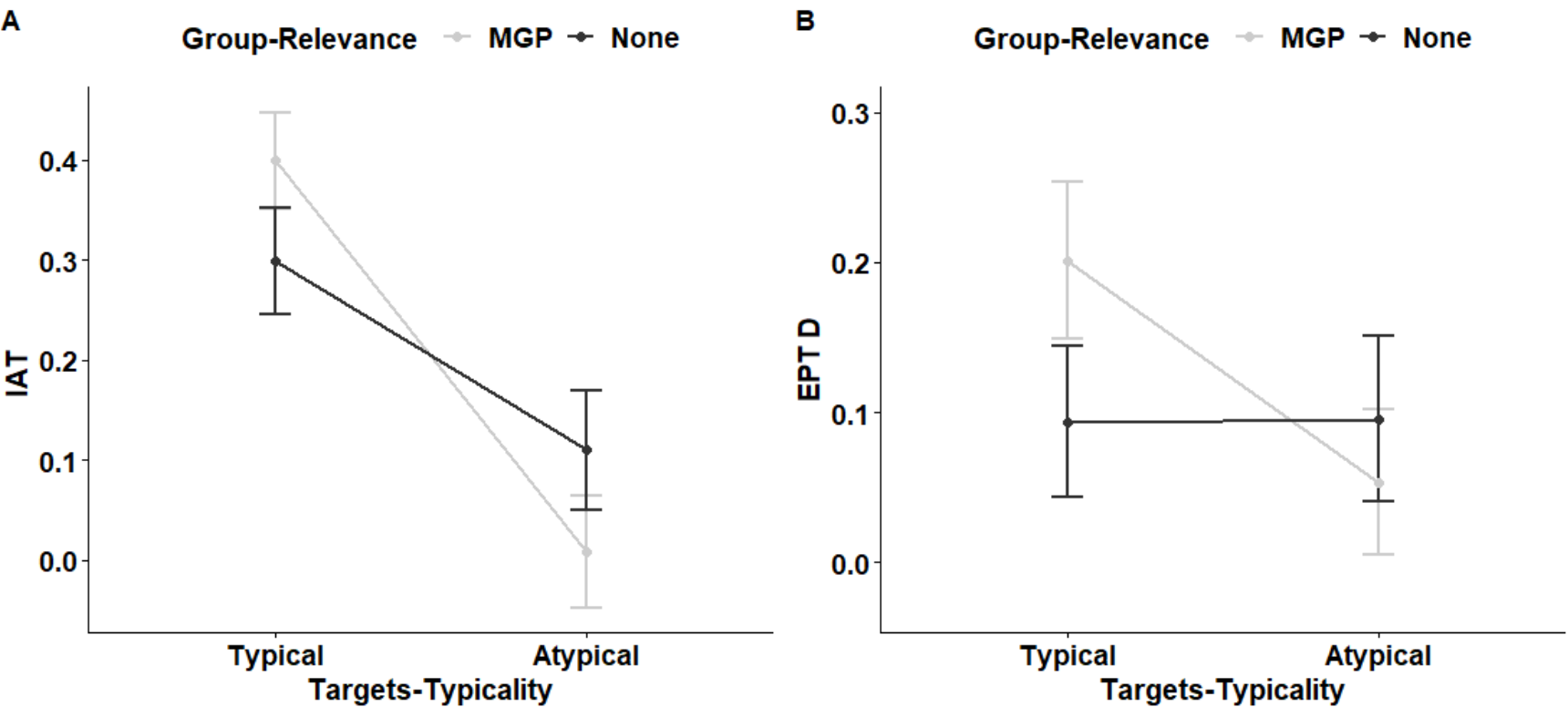

Figure 5. IAT (A) and EPT (B) scores (preference for the positively portrayed individual) as a function of targets-type and the relevance of the group to the participant in Experiments 6 and 7.

\section{Discussion}


Experiments 6 and 7 found that the motivational (social) relevance of the judged groups whether the participants belonged (or did not belong) to one of the groups - moderated perceivers' directly and indirectly measured evaluation of individuals from those groups. However, that factor moderated different effects on each type of evaluation measure: Relevance to the group led to stronger effect of the individuating information on the self-reported evaluations and a stronger effect of the group information on the preferences measured with the IAT and EPT. We speculate that the moderating effect of self-relevance on self-reported evaluation reflected an increased attention to the evaluative information. The moderating effect of social-relevance on the effect of group information on the IAT and the EPT reflects an ingroup bias, in favor of one's group members over outgroup members. The meta-analysis of the results of the basic paradigm and the results of Experiment 5 already suggested that automatic evaluation of individual group members might be more sensitive to group information than deliberate evaluation. Experiments 6 and 7 provide further support to that conclusion, showing that when a negatively-portrayed person belonged to the participant's group, there was no longer a significant preference for the positivelyportrayed individual (outgroup member) over the negatively-portrayed ingroup member. Whereas deliberate evaluation successfully ignored group membership in all conditions of Experiments 6 and 7, the automatic evaluation was clearly sensitive to that information, especially when the group information indicated whether the target belonged to the same group as the participant.

\section{General Discussion}

In seven main experiments and 12 auxiliary experiments, we used an impression formation paradigm to investigate the effect of group information and individuating information on people's directly and indirectly measured evaluations of individual members of novel social groups. Across the two types of measures, we found that individuating information had a large influence on participants' evaluations while group information had a much smaller influence. Participants showed a preference for a target individual who performed positive behaviors over a target individual who performed negative behaviors, even when the groups of the two individuals had a 
valence opposite to the valence of the individuals. Both directly and indirectly measured (with the IAT and the EPT, yet not with SPF and AMP in Part 1) evaluations also showed some sensitivity to information about the groups: the preference for the individual who behaved positively was slightly larger when the individual belonged to a positively-valenced group, than when the individual belonged to a negatively-valenced group. Yet, that influence was much smaller, compared with that of the individuating information.

In a comparison of direct (self-report) and indirect measures, although both measures were more sensitive to individuating information than to group information, this difference was larger in direct measures (a meta-analysis estimate of Cohen's $d=1.78$ [CI 95\% 1.66-1.90] for the individuating information effect, and Cohen's $d=0.13$ [CI 95\% 0.04-0.22] for the group information effect) than in indirect measures (a meta-analysis estimate of Cohen's $d=0.42[C I 95 \%$ 0.34-0.49] for the individuating information effect and Cohen's $d=0.23$ [CI 95\% 0.14-0.33] for the group information effect). This discrepancy between direct and indirect measures in their relative sensitivity to the two sources of evaluative information was statistically significant, in all the experiments (Table 3). Although the IAT influenced the meta-analysis more than the other indirect measures, and only the IAT and the EPT showed a significant effect of group information in a single study, the direct test of discrepancy detailed in Table 3 found a significant discrepancy between the self-report and all the indirect measures that we used in this research, including the SPF (Experiment 2) and the AMP (Experiment 3).

As a whole, the results of Part 1 of the present research suggest that although both automatic and deliberate evaluation of individual group members are largely sensitive to specific individuating information about the judged individuals and, to a lesser extent, to the group membership of these individuals, there is also a clear discrepancy in the relative influence of these two sources of information on deliberate versus automatic evaluation: in comparison to automatic evaluation, deliberate evaluation is more sensitive to individuating information relatively to group information. 
In Part 2 of the present research, we tested variants of the basic paradigm, each added to the minimalistic procedure one factor that might be common in the judgment of individual group members in everyday life. In Experiment 4, we found that adding information of consistent valence about the groups did not strengthen the effect of group information on evaluation. In Experiment 5, we found that emphasizing the target individual's group membership upon judgment increased the effect of group information on the IAT scores but not on self-reported evaluation. In Experiments 6 and 7, we found that assigning the participants to the positively-portrayed group increased the effect of group information on evaluation when it was measured indirectly with the IAT and the EPT, but not when it was measured directly with a self-report questionnaire. Specifically, participants' IAT scores no longer suggested an automatic preference for the positively-portrayed target over the negatively-portrayed target when the negatively-portrayed target belonged to the participant's ingroup. In that condition, the EPT score showed a very slight preference for the positivelyportrayed outgroup member over the negatively-portrayed ingroup member, significantly smaller than the automatic preference suggested by the EPT scores in the parallel condition that did not assign the participants to any of the groups.

Interestingly, the self-reported evaluation was also sensitive to the participants' relation to the groups, but quite differently. Self-reportedly, participants' preference for the positivelyportrayed individual over the negatively-portrayed individual was stronger when the participant belonged to one of the groups than when the participant was not assigned to any of the groups, regardless of the individuals' specific group membership (i.e., even when the negatively-portrayed individual belongs to the participants' group). The results of Experiments 4-7 strengthen the possibility that, while automatic evaluation of individual group members is not substantially more biased than deliberate evaluation by group information, two important factors may increase that discrepancy in everyday life - the salience of group membership upon judgment and the relevance of the groups to the perceiver.

\section{Theoretical Implications}




\section{Information About the Group Is Not Inherently the Most Influential on Automatic Evaluation}

In our basic paradigm, we tried to provide the minimal information needed for forming an accurate judgment of the whole group and of each of the group's individual members. Our purpose was to test whether these conditions are sufficient to cause prejudice in the automatic judgment of the individual members of the group. We found that judgment of individual group members was only slightly biased by the group information. The most compelling evidence for the dominance of individuating information on automatic and deliberate evaluation comes from Experiment 4: even when we increased the amount and consistency of the information about the group (seven out of eight individuals in each group were characterized by behavior of the same valence), the individuating information was still the dominant influence on all evaluation measures. This finding increases our confidence in the conclusion that automatic evaluation is not inherently more sensitive to group information than to individuating information.

Based on early theories of person perception (e.g., Brewer, 1988; Fiske \& Neuberg, 1990), it is plausible to hypothesize that automatic evaluation of individual group members would rely mostly on group information. Although these theories never made explicit hypotheses regarding automatic (as opposed to deliberate) evaluation, they contended that basing judgment on group information is less effortful and more resources-efficient than judgment that is based on individuating information. Moreover, these theories suggest that reliance on individuating information depends on motivation and cognitive resources, which seems more relevant for deliberate, rather than automatic evaluation. This prediction is also in line with research showing that repeated pairing of certain group features with valence leads to the automatic activation of valence upon exposure to these features, independently of any other source of evaluative information (Livingston \& Brewer, 2002). Further, in some previous research, the automatic judgment of individual members of existing social groups was determined by group information rather than by individuating information (Cao \& Banaji, 2016; Navon et al., 2021). Because our 
minimalistic paradigm did not replicate these results, we suggest that the previous findings were caused by factors other than mere knowledge of the target individuals' group membership.

The present findings are more compatible with later theories of social judgment that suggested that group information does not necessarily take primacy in people's evaluations, compatible with the possibility that deliberate judgment of the target individuals could be automatized and activated upon judgment (De Houwer, 2014, 2018; Kunda \& Thagard, 1996; Kurdi \& Banaji, 2017; Kurdi \& Dunham, 2020; Mandelbaum, 2016; Monroe et al., 2018; Van Dessel et al., 2019). Our findings suggest that automatic evaluation of individual group members is sensitive to information diagnosticity. In Experiments S6 and S7 in the supplement, we replicated with our minimal paradigm the finding that, in the absence of diagnostic individuating information, participants' judgment reflects the information known about the social groups, which was the most (and only) diagnostic information available. However, when the more diagnostic individuating information was present, it dominated participants' automatic and deliberate evaluations (see also Rubinstein et al., 2018).

\section{Group Information Influences Automatic Evaluation More than Deliberate Evaluation}

Although we did not find evidence that group information is ever the dominant influence on deliberate or automatic evaluation, we found ample evidence that the sensitivity to group information relatively to individuating information was somewhat larger on the indirect measures that we used to measure automatic evaluation than on the direct measures that we used to measure deliberate evaluation. These results suggest that, relatively to deliberate evaluation, group information biases automatic evaluation more biased than deliberate evaluation (relatively to the strength of the effect of individuating information on evaluation). In other words, automatic prejudice is stronger than deliberate prejudice. This finding is compatible with the perspective that attributes prejudice predominantly to unintentional and possibly uncontrolled judgment processes (Dovidio \& Gaertner, 2004; Hardin \& Banaji, 2013; Lai et al., 2013; Levy \& Banaji, 2002; Payne et al., 2010). 
The present findings suggest that the discrepancy between deliberate and automatic prejudice occurs even in a minimal procedure that only provided evaluative information and group information about previously unknown individuals. That procedure was devoid of many aspects that characterize social groups that people know in their everyday life (e.g., beliefs about how the history of the group or other features of the group would affect the typical attributes of its individual members, or the group affiliation of the perceiver). And yet, the mere grouping of target individuals together was enough to cause more prejudice in automatic than in deliberate judgment.

One possible reason for the discrepancy that we found in the influence of group information on automatic vs. deliberate evaluation is that participants spontaneously use group information when judging individual group members; but, with effort, they reject group information as a relevant source. According to this account, because automatic evaluation does not provide opportunity to consider and reject spontaneous influences, it was more sensitive to group information than deliberate evaluation was. This possibility is compatible with the prevalent notion that deliberate evaluation is less prejudiced and more egalitarian than automatic evaluation because it is based on processes that can alter the spontaneously activated evaluation (e.g., Gawronski \& Bodenhausen, 2006, 2007, 2011; Petty et al., 2007).

\section{The Factors that Increase the Effect of Group Information on Automatic Evaluation}

Amount of information about the group. In our basic impression formation paradigm, we only provided information about individuals and grouped them into two groups. Starting with such a minimal paradigm allows adding specific factors and testing how each of them influences the judgment of individual group members. In the present research, we tested three such factors: the amount of information about the group, the salience of the group membership upon judgment, and the relevance of the groups to the perceiver. We found no evidence that increasing the number of typical group members influences the judgment of the atypical group members. This result should be interpreted with caution because it is a null result. Yet, if the result does reflect a general rule, it might suggest that the dominance of the individuating information is unrelated to how strong the 
information about the group is. Perhaps people form impressions of other people based on the most diagnostic, individuating information about the specific person they judge, and any effect of the group is not due to the strength of the judgment of the whole group, or the average group member. Notably, the effect of the amount of information about the group on the judgment of individual group members was difficult to test in previous research that used existing social groups.

Researchers cannot control the information about existing social groups. Different people probably learned different pieces of information about existing social groups, and different amounts of information. This, in turn, could lead to an asymmetry between group information and individuating information about members of existing social groups. This asymmetry is easy to eliminate with novel social groups, as we did in the present research.

Group salience. The second potential moderator that we tested in the present research was the salience of the group membership. Previous research with pre-existing social groups found that the influence of group information on automatic (but not deliberate) evaluation of famous members of known groups (Black and White people) was larger when the stimuli that represented the social groups were photos of the target people than when they were the name of the targets (Navon et al., 2021). That effect might reflect stronger influence of group information on the automatic judgment of individual group members when the group membership is more accessible upon judgment. The present research is compatible with that hypothesis: the influence of group information on the evaluation measured by the IAT was the largest when the category labels in the IAT were textual but referred to the individuals as members of their group (e.g., the Baunif), rather than used the photo of the specific individual (the stimuli of the category items were the photos of the target individuals). In contrast, referring to the target individuals by their group membership (instead of only showing their photos) had no effect on people's self-reported evaluation of those targets.

A possible reason for the discrepancy in the effect of group salience on automatic vs. deliberate evaluation might be that, upon deliberate evaluation, people had ample cognitive resources, intention, and time to ignore the spontaneous evaluation that the salient group 
membership might elicit, and to intentionally use the information remembered about the specific target individual. In contrast, when completing the IAT, the salience of group membership might increase the likelihood (or strength) of the spontaneous activation of the evaluation of the group. Because performing the IAT does not require intentional evaluation of the groups, it is plausible that participants did not recruit any deliberate, cognitively effortful, processes that would reject the influence of the spontaneous evaluation of the group, and would allow only the relevant and diagnostic individuating information about the specific person to influence the evaluation. Inasmuch as performance in the IAT reflects automatic evaluation in other contexts, our results suggest that the salience of group membership increases the likelihood of automatic but not of deliberate prejudice.

Motivational relevance. The third moderator that we investigated in the present research was the motivational relevance of the group to the perceiver. People belong to certain social groups (i.e., their ingroups), and not to others (the outgroups). The motivation to feel good about oneself, among other possible factors (Hogg \& Abrams, 1988; Park et al., 2008, 2008; Sidanius \& Pratto, 1999; Tajfel et al., 1979; Turner, 1975; Van Vugt \& Schaller, 2008; Yamagishi et al., 1999), drives people to favor members of their own group over members of other groups (Brewer, 1979; Levine \& Campbell, 1972; Mullen et al., 1992; Oakes \& Turner, 1980; Tajfel, 1982). We took advantage of our impression formation paradigm, to examine whether sharing a group membership with one of the two target individuals would increase the effect of group information on evaluation, relatively to the effect of individuating information. We found that when the groups were relevant to the participant because they were the participant's ingroup and outgroup, the effect of group information on the evaluation of individual group members increased when the evaluation was measured indirectly by measures developed to capture automatic evaluation (the IAT and EPT), but not when measured directly with a self-report measure.

As with group salience, the (in)ability to reject or overcome spontaneous evaluative processes might explain the discrepancy in the effect of group relevance on automatic vs. deliberate 
evaluation. People's high motivation to reach self-enhancing conclusions (my group member is better than a member of another group) might conflict with their motivation to provide accurate and fair judgment (Kunda, 1990). With intention and effort, people can ignore group relevance when they judge target individuals deliberately, but the same effortful processes might not be as easily deployed when the evaluation is relatively unintentional and cannot recruit ample cognitive resources.

Together, our findings suggest that previously found effects of group information on automatic judgment of individuals (Cao \& Banaji, 2016; Navon et al., 2021) mostly reflect the influence of factors other than mere knowledge that the target individual belongs to a positively or negatively evaluated group. Here, we found two such factors: group salience and group relevance. In the supplement, we report experiments that tested more factors: we did not find evidence for a moderating effect of practice in associating the group members with their groups (Experiment S4) and found inconclusive evidence for providing general information about the group, rather than only the individual group members (Experiments S8-S11). We continue to study these factors and other factors that might moderate the effect of group information on evaluation.

\section{Implications for the Study of Indirect Measures of Judgment}

As summarized briefly in the introduction, there is much evidence that the indirect measures that we used are sensitive to automatic evaluative processes. However, for each measure, there is also evidence that the measure is also sensitive to factors that are not automatic evaluation. Aware of these limitations, we used four different indirect measures that were developed to measure automatic evaluation. Findings the same results with all measures would decrease the range of alternative accounts that attribute the results to constructs other than automatic evaluation. Finding different results with different measures would add knowledge about possible differences in the constructs captured by each measure.

In the present research, the scores of all four measures were sensitive to the individuating information. In all four measures, the pattern of the results was compatible with a slight effect of 
group information on judgment, but that effect was a bit stronger with the IAT and EPT relative to SPF and AMP, in which the effect did not reach significance. Finally, the finding that assigning the participants to one of the groups increases the effect of group information on indirectly measured evaluation was found with the IAT and replicated with the EPT. The relative consistency of the findings with the various measures is compatible with the possibility that all these measures are sensitive to the same construct. Because these measures were developed to measure automatic evaluation and, currently, there is no published research that attribute results found with two of these measures to one construct other than automatic evaluation, the most likely conclusion is that our results pertain to automatic evaluation. Yet, future evidence could change that conclusion, and the present results might provide clues for developing hypotheses about alternative constructs.

More generally, Experiments 6 and 7 are of the very few instances of impression formation research that found, with more than one indirect measure, a discrepancy between self-reported and indirect measured evaluation (see also Van Dessel et al., 2020; and Moran \& Bar-Anan, 2013, which was later challenged by Bading et al., 2020). As such, further research could use the paradigm of these experiments for improving the understanding of automatic evaluation and its supposed measures. For example, it would be interesting to test a host of different indirect measures and see which of them replicates the same discrepancy. Such an investigation could provide further clues regarding the construct(s) that the different indirect measures capture.

\section{Limitations and Future Directions}

Although we replicated our effects across various stimuli, indirect measures and some methodological variations of the paradigm, it is important to note that all experiments used the same basic attitude induction paradigm and one measure of self-reported evaluation. Thus, a generalization of our findings to other paradigms and self-report measures would be of value, as it would strengthen the confidence that our findings do not depend on specific properties of our paradigm. The replication of the previous known effects of group information when diagnostic individuating effect was not available (Experiments S6-S7), and by the factors we tested in 
Experiments 5-7 mitigates this threat, as these results are in line with theory and research about evaluation. Further support comes from our findings from Experiments S8-S11, that employed a more direct induction of attitudes toward the two groups, yielding no consistent advantage of that information in automatic judgment. Still, direct tests of the generalization of our findings to other paradigms would provide better evidence for their pervasiveness.

Beyond the generalization to another paradigm, a more complete examination of the effect of individuating information and group information on the judgment of individual group members would also include a test of the effect of these two sources of information on judgment related to specific attributes of individual group members (i.e., stereotypes). While the present research found that prejudice is applied only to a small extent in the presence of more diagnostic information, that would not be necessarily the case for stereotypes, as they can operate independently of prejudice (Amodio, 2019; Amodio \& Devine, 2006).

Finally, it would be informative to investigate the reasons for the larger sensitivity to group information on the IAT and EPT relative to the SPF and AMP. An initial step would be to conduct a direct replication of our basic paradigm with these four measures within the same experiment, to control for effects that are unrelated to the measures. Then, future research could test possible explanations for the differences between these measures, such as their sensitivity to automatic judgment processes, and the specific automaticity feature(s) that they capture. 


\section{Conclusion}

Theory and research suggest that automatic evaluation of individual group members is determined mostly by evaluative information about the individuals' groups, ignoring any unique information known about the evaluated individual. Yet, our research suggests that information about the individual strongly influences automatic evaluation, while the influence of group information is small. Nevertheless, compared with its effect on deliberate evaluation, group information has a slightly larger influence on automatic evaluation. The discrepancy between deliberate and automatic evaluations in their sensitivity to group information increases in situations that often characterize the judgment of individual group members in everyday life.

\section{Context of the Research}

Implicit bias, that is, a bias in the way people judge others unintentionally, is a widely studied phenomenon, across research fields and cultures. The prevalent notion among researchers is that, by default, spontaneously and without intention, people judge others by their group membership, even when they do not endorse the group-related information and think that individuals' personal characteristics are more diagnostic of their character. Yet, empirical research about the automatic judgment of individuals, rather than whole groups, is surprisingly scarce. The present research is a part of a larger program of research that aims to thoroughly investigate the relative influence of group information and individuating information on automatic judgment, across various judgment contexts. This program of research has largely demonstrated that group information has a robust and consistent influence on automatic judgments of individuals, even though the perceivers are provided with more diagnostic individuating information about the target individuals (Navon, 2021; Navon \& Bar-Anan, 2022; Navon et al., 2021). Importantly, while prior work in this program has focused on judgments of individuals from existing social groups, the present work extends that research by examining novel social groups, thus allowing better control of the content of the two sources of information. 


\section{References}

Allport, G. W. (1954). The nature of prejudice. Addison-Wesley.

Amodio, D. M. (2019). Social Cognition 2.0: An interactive memory systems account. Trends in Cognitive Sciences, 23(1), 21-33. https://doi.org/10.1016/j.tics.2018.10.002

Amodio, D. M., \& Devine, P. G. (2006). Stereotyping and evaluation in implicit race bias: Evidence for independent constructs and unique effects on behavior. Journal of Personality and Social Psychology, 91(4), 652-661. https://doi.org/10.1037/0022-3514.91.4.652

Amodio, D. M., \& Mendoza, S. A. (2010). Implicit intergroup bias: Cognitive, affective, and motivational underpinnings. In Handbook of implicit social cognition: Measurement, theory, and applications. (pp. 353-374). The Guilford Press.

Asendorpf, J. B., Banse, R., \& Mücke, D. (2002). Double dissociation between implicit and explicit personality self-concept: The case of shy behavior. Journal of Personality and Social Psychology, 83(2), 380-393. https://doi.org/10.1037/0022-3514.83.2.380

Ashburn-Nardo, L., Voils, C. I., \& Monteith, M. J. (2001). Implicit associations as the seeds of intergroup bias: How easily do they take root? Ournal of Personality and Social Psychology, 81(5), 789-799. https://psycnet.apa.org/doi/10.1037/0022-3514.81.5.789

Axt, J. R., Bar-Anan, Y., \& Vianello, M. (2020). The relation between evaluation and racial categorization of emotional faces. Social Psychological and Personality Science, 11(2), 196-206. https://doi.org/10.1177/1948550619848000

Axt, J. R., Ebersole, C. R., \& Nosek, B. A. (2014). The rules of implicit evaluation by race, religion, and age. Psychological Science, 25(9), 1804-1815.

Axt, J. R., \& Lai, C. K. (2019). Reducing discrimination: A bias versus noise perspective. Journal of Personality and Social Psychology, 117(1), 26-49.

Bading, K., Stahl, C., \& Rothermund, K. (2020). Why a standard IAT effect cannot provide evidence for association formation: The role of similarity construction. Cognition and Emotion, 34(1), 128-143. 
Banaji, M. R., \& Bhaskar, R. (2000). Implicit stereotypes and memory: The bounded rationality of social beliefs. In DL Schacter \& E. Scarry (Eds.), Memory, Brain, and Belief.

Banse, R., Seise, J., \& Zerbes, N. (2001). Implicit attitudes towards homosexuality: Reliability, validity, and controllability of the IAT. Zeitschrift Für Experimentelle Psychologie, 48(2), $145-160$.

Bar-Anan, Y., \& Nosek, B. A. (2012). Reporting intentional rating of the primes predicts priming effects in the affective misattribution procedure. Personality and Social Psychology Bulletin, 38(9), 1194-1208.

Bar-Anan, Y., \& Nosek, B. A. (2014). A comparative investigation of seven indirect attitude measures. Behavior Research Methods, 46(3), 668-688. https://doi.org/10.3758/s13428013-0410-6

Bar-Anan, Y., Nosek, B. A., \& Vianello, M. (2009). The sorting paired features task: A measure of association strengths. Experimental Psychology, 56(5), 329-343.

Bargh, J. (1994). The four horsemen of automaticity: Awareness, efficiency, intentions and control. In R. Wyer \& T. Srull (Eds.), Handbook of Social Cognition (p. 1040). Lawrence Erlbaum. Baugh, F. (2002). Correcting Effect Sizes for Score Reliability: A Reminder that Measurement and Substantive Issues are Linked Inextricably. Educational and Psychological Measurement, 62(2), 254-263. https://doi.org/10.1177/0013164402062002004

Bernstein, M. J., Young, S. G., \& Hugenberg, K. (2007). The cross-category effect: Mere social categorization is sufficient to elicit an own-group bias in face recognition. Psychological Science, 18(8), 706-712.

Blair, I. V. (2002). The malleability of automatic stereotypes and prejudice. Personality and Social Psychology Review, 6(3), 242-261.

Blair, I. V., \& Banaji, M. R. (1996). Automatic and controlled processes in stereotype priming. Journal of Personality and Social Psychology, 70(6), 1142-1163. https://doi.org/10.1037/0022-3514.70.6.1142 
Bodenhausen, G. V., Kang, S. K., \& Peery, D. (2012). Social categorization and the perception of social groups. In The SAGE Handbook of Social Cognition The SAGE handbook of social cognition (pp. 311-329). SAGE Publications Ltd.

https://doi.org/10.4135/9781446247631.n16

Bodenhausen, G. V., \& Macrae, C. N. (1998). Stereotype activation and inhibition. In Stereotype activation and inhibition. (pp. 1-52). Lawrence Erlbaum Associates Publishers.

Borenstein, M., Hedges, L. V., Higgins, J. P. T., \& Rothstein, H. R. (2009). Introduction to MetaAnalysis John Wiley \& Sons. Ltd, Chichester, UK.

Brewer, M. (1988). A dual process model of impression formation. TK Srull, RS Wyer, Jr., eds. Advances in Social Cognition, Vol. 1. Erlbaum, Hillsdale, NJ.

Brewer, M. B. (1979). In-group bias in the minimal intergroup situation: A cognitive-motivational analysis. Psychological Bulletin, 86(2), 307-324.

Brewer, M. B., \& Silver, M. (1978). Ingroup bias as a function of task characteristics. European Journal of Social Psychology, 8(3), 393-400.

Brown-Iannuzzi, J. L., Cooley, E., McKee, S. E., \& Hyden, C. (2019). Wealthy Whites and poor Blacks: Implicit associations between racial groups and wealth predict explicit opposition toward helping the poor. Journal of Experimental Social Psychology, 82, 26-34.

Cameron, C. D., Brown-Iannuzzi, J. L., \& Payne, B. K. (2012). Sequential priming measures of implicit social cognition: A meta-analysis of associations with behavior and explicit attitudes. Personality and Social Psychology Review, 16(4), 330-350.

Cao, J., \& Banaji, M. R. (2016). The base rate principle and the fairness principle in social judgment. Proceedings of the National Academy of Sciences, 113(27), 7475-7480. https://doi.org/10.1073/pnas.1524268113

Castelli, L., Zogmaister, C., Smith, E. R., \& Arcuri, L. (2004). On the automatic evaluation of social exemplars. Journal of Personality and Social Psychology, 86(3), 373-387. https://doi.org/10.1037/0022-3514.86.3.373 
Charlesworth, T. E., \& Banaji, M. R. (2019). Patterns of implicit and explicit attitudes: I. Long-term change and stability from 2007 to 2016. Psychological Science, 30(2), 174-192.

Chun, W. Y., \& Kruglanski, A. W. (2006). The role of task demands and processing resources in the use of base-rate and individuating information. Journal of Personality and Social Psychology, 91(2), 205-217.

Corneille, O., \& Hütter, M. (2020). Implicit? What do you mean? A comprehensive review of the delusive implicitness construct in attitude research. Personality and Social Psychology Review, 24(3), 212-232. https://doi.org/10.1177/1088868320911325

Dasgupta, N. (2004). Implicit ingroup favoritism, outgroup favoritism, and their behavioral manifestations. Social Justice Research, 17(2), 143-169.

Dasgupta, N., McGhee, D. E., Greenwald, A. G., \& Banaji, M. R. (2000). Automatic preference for White Americans: Eliminating the familiarity explanation. Journal of Experimental Social Psychology, 36(3), 316-328. https://doi.org/10.1006/jesp.1999.1418

De Houwer, J. (2006). What are implicit measures and why are we using them. In The handbook of implicit cognition and addiction (pp. 11-28). Sage.

De Houwer, J. (2014). A propositional model of implicit evaluation. Social and Personality Psychology Compass, 8(7), 342-353. https://doi.org/10.1111/spc3.12111

De Houwer, J. (2018). Propositional models of evaluative conditioning. Social Psychological Bulletin, 13(3), 1-21.

DeSteno, D., Dasgupta, N., Bartlett, M. Y., \& Cajdric, A. (2004). Prejudice from thin air: The effect of emotion on automatic intergroup attitudes. Psychological Science, 15(5), 319-324.

Devine, P. G. (1989). Stereotypes and prejudice: Their automatic and controlled components. Journal of Personality and Social Psychology, 56(1), 5-18. https://doi.org/10.1037/00223514.56.1.5 
Devine, P. G., Monteith, M. J., Zuwerink, J. R., \& Elliot, A. J. (1991). Prejudice with and without compunction. Journal of Personality and Social Psychology, 60(6), 817-830. https://doi.org/10.1037/0022-3514.60.6.817

Devine, P. G., Plant, E. A., Amodio, D. M., Harmon-Jones, E., \& Vance, S. L. (2002). The regulation of explicit and implicit race bias: The role of motivations to respond without prejudice. Journal of Personality and Social Psychology, 82(5), 835-848. https://doi.org/10.1037//0022-3514.82.5.835

Dovidio, J. F., \& Gaertner, S. L. (2004). Aversive racism. Advances in Experimental Social Psychology, Vol. 36., 1-52. https://doi.org/10.1016/S0065-2601(04)36001-6

Dovidio, J. F., Kawakami, K., Johnson, C., Johnson, B., \& Howard, A. (1997). On the nature of prejudice: Automatic and controlled processes. Journal of Experimental Social Psychology, 33(5), 510-540. https://doi.org/10.1006/jesp.1997.1331

Dunham, Y. (2013). Balanced identity in the minimal groups paradigm. PloS One, 8(12), e84205.

Egloff, B., \& Schmukle, S. C. (2002). Predictive validity of an implicit association test for assessing anxiety. Journal of Personality and Social Psychology, 83(6), 1441-1455. https://doi.org/10.1037/0022-3514.83.6.1441

Fazio, R. H., Jackson, J. R., Dunton, B. C., \& Williams, C. J. (1995). Variability in automatic activation as an unobtrusive measure of racial attitudes: A bona fide pipeline? Journal of Personality and Social Psychology, 69(6), 1013-1027. https://doi.org/10.1037/00223514.69.6.1013

Fazio, R. H., Sanbonmatsu, D. M., Powell, M. C., \& Kardes, F. R. (1986). On the automatic activation of attitudes. Journal of Personality and Social Psychology, 50(2), 229-238.

Ferguson, C. K., \& Kelley, H. H. (1964). Significant factors in overevaluation of own-group's product. The Journal of Abnormal and Social Psychology, 69(2), 223-228. https://psycnet.apa.org/doi/10.1037/h0046572 
Fiske, S. T., \& Neuberg, S. L. (1990). A continuum of impression formation, from category-based to individuating processes: Influences of information and motivation on attention and interpretation. In Advances in experimental social psychology (Vol. 23, pp. 1-74). Elsevier.

Fiske, S. T., \& Taylor, S. E. (1991). Social cognition. Mcgraw-Hill Book Company.

Friese, M., Hofmann, W., \& Wänke, M. (2008). When impulses take over: Moderated predictive validity of explicit and implicit attitude measures in predicting food choice and consumption behaviour. British Journal of Social Psychology, 47(3), 397-419.

Gawronski, B., \& Bodenhausen, G. V. (2006). Associative and propositional processes in evaluation: An integrative review of implicit and explicit attitude change. Psychological Bulletin, 132(5), 692-731. https://doi.org/10.1037/0033-2909.132.5.692

Gawronski, B., \& Bodenhausen, G. V. (2007). Unraveling the processes underlying evaluation: Attitudes from the perspective of the APE model. Social Cognition, 25(5), 687-717.

Gawronski, B., \& Bodenhausen, G. V. (2011). The Associative-Propositional Evaluation Model. In Advances in Experimental Social Psychology (Vol. 44, pp. 59-127). Elsevier. https://doi.org/10.1016/B978-0-12-385522-0.00002-0

Gawronski, B., \& Bodenhausen, G. V. (2018). Evaluative conditioning from the perspective of the associative-propositional evaluation model. Social Psychological Bulletin, 13(3), 1-33.

Gawronski, B., \& De Houwer, J. (2014). Implicit measures in social and personality psychology. In Handbook of research methods in social and personality psychology (pp. 283-310). Cambridge University Press.

Gawronski, B., De Houwer, J., \& Sherman, J. (in press). Twenty-Five Years of Research Using Implicit Measures. Social Cognition.

Gawronski, B., \& Hahn, A. (2019). Implicit measures: Procedures, use, and interpretation. Measurement in Social Psychology., 29-55.

Greenwald, A. G., McGhee, D. E., \& Schwartz, J. L. K. (n.d.). Measuring Individual Differences in Implicit Cognition: The Implicit Association Test. 17. 
Greenwald, A. G., McGhee, D. E., \& Schwartz, J. L. K. (1998). Measuring individual differences in implicit cognition: The implicit association test. Journal of Personality and Social Psychology, 74(6), 1464-1480. https://doi.org/10.1037/0022-3514.74.6.1464

Greenwald, A. G., \& Nosek, B. A. (2001). Health of the Implicit Association Test at age 3. Zeitschrift Für Experimentelle Psychologie.

Greenwald, A. G., Nosek, B. A., \& Banaji, M. R. (2003). Understanding and using the Implicit Association Test: I. An improved scoring algorithm. Journal of Personality and Social Psychology, 85(2), 197-216. https://doi.org/10.1037/0022-3514.85.2.197

Hardin, C. D., \& Banaji, M. R. (2013). The nature of implicit prejudice. In The behavioral foundations of public policy (pp. 13-31). Princeton University Press.

Hermans, D., Crombez, G., \& Eelen, P. (2000). Automatic attitude activation and efficiency: The fourth horseman of automaticity. Psychologica Belgica, 40(1), 3-22.

Hofmann, W., Gschwendner, T., Friese, M., Wiers, R. W., \& Schmitt, M. (2008). Working Memory Capacity and Self-Regulatory Behavior: Toward an Individual Differences Perspective on Behavior Determination by Automatic Versus Controlled Processes. Journal of Personality and Social Psychology, 95(4), 962-977.

Hofmann, W., Rauch, W., \& Gawronski, B. (2007). And deplete us not into temptation: Automatic attitudes, dietary restraint, and self-regulatory resources as determinants of eating behavior. Journal of Experimental Social Psychology, 43(3), 497-504.

Hogg, M. A., \& Abrams, D. (1988). Social identifications: A social psychology of intergroup relations and group processes. Taylor \& Frances/Routledge.

Ito, T. A., Friedman, N. P., Bartholow, B. D., Correll, J., Loersch, C., Altamirano, L. J., \& Miyake, A. (2015). Toward a comprehensive understanding of executive cognitive function in implicit racial bias. Journal of Personality and Social Psychology, 108(2), 187-218. https://doi.org/10.1037/a0038557 
Ito, T. A., \& Urland, G. R. (2003). Race and gender on the brain: Electrocortical measures of attention to the race and gender of multiply categorizable individuals. Journal of Personality and Social Psychology, 85(4), 616-626. https://doi.org/10.1037/0022-3514.85.4.616

Ito, T. A., \& Urland, G. R. (2005). The influence of processing objectives on the perception of faces: An ERP study of race and gender perception. Cognitive, Affective, \& Behavioral Neuroscience, 5(1), 21-36. https://doi.org/10.3758/CABN.5.1.21

Kawakami, K., \& Dovidio, J. F. (2001). The reliability of implicit stereotyping. Personality and Social Psychology Bulletin, 27(2), 212-225.

Kim, D.-Y. (2003a). Voluntary controllability of the implicit association test (IAT). Social Psychology Quarterly, 83-96.

Kim, D.-Y. (2003b). Voluntary Controllability of the Implicit Association Test (IAT). Social Psychology Quarterly, 66(1), 83-96. JSTOR. https://doi.org/10.2307/3090143

Kirby, S., Cornish, H., \& Smith, K. (2008). Cumulative cultural evolution in the laboratory: An experimental approach to the origins of structure in human language. Proceedings of the National Academy of Sciences, 105(31), 10681-10686.

Klauer, K. C., \& Teige-Mocigemba, S. (2007). Controllability and resource dependence in automatic evaluation. Journal of Experimental Social Psychology, 43(4), 648-655.

Kruglanski, A. W., \& Webster, D. M. (1991). Group members' reactions to opinion deviates and conformists at varying degrees of proximity to decision deadline and of environmental noise. Journal of Personality and Social Psychology, 61(2), 212-225.

Kunda, Z. (1990). The case for motivated reasoning. Psychological Bulletin, 108(3), 480-498.

Kunda, Z., Davies, P. G., Adams, B. D., \& Spencer, S. J. (2002). The dynamic time course of stereotype activation: Activation, dissipation, and resurrection. Journal of Personality and Social Psychology, 82(3), 283-299. https://doi.org/10.1037/0022-3514.82.3.283 
Kunda, Z., \& Sherman-Williams, B. (1993). Stereotypes and the construal of individuating information. Personality and Social Psychology Bulletin, 19(1), 90-99. https://doi.org/10.1177/0146167293191010

Kunda, Z., \& Spencer, S. J. (2003). When do stereotypes come to mind and when do they color judgment? A goal-based theoretical framework for stereotype activation and application. Psychological Bulletin, 129(4), 522-544. https://doi.org/10.1037/0033-2909.129.4.522

Kunda, Z., \& Thagard, P. (1996). Forming impressions from stereotypes, traits, and behaviors: A parallel-constraint-satisfaction theory. Psychological Review, 103(2), 284-308.

Kurdi, B., \& Banaji, M. R. (2017). Repeated evaluative pairings and evaluative statements: How effectively do they shift implicit attitudes? Journal of Experimental Psychology: General, $146(2), 194-213$.

Kurdi, B., \& Dunham, Y. (2020). Propositional accounts of implicit evaluation: Taking stock and looking ahead. Social Cognition, 38(Supplement), s42-s67.

Kurdi, B., Seitchik, A. E., Axt, J. R., Carroll, T. J., Karapetyan, A., Kaushik, N., Tomezsko, D., Greenwald, A. G., \& Banaji, M. R. (2019). Relationship between the Implicit Association Test and intergroup behavior: A meta-analysis. American Psychologist, 74(5), 569-586. https://doi.org/10.1037/amp0000364

Lai, C. K., Hoffman, K. M., \& Nosek, B. A. (2013). Reducing implicit prejudice. Social and Personality Psychology Compass, 7(5), 315-330. https://doi.org/10.1111/spc3.12023

Lakens, D. (2013). Calculating and reporting effect sizes to facilitate cumulative science: A practical primer for t-tests and ANOVAs. Frontiers in Psychology, 4. https://doi.org/10.3389/fpsyg.2013.00863

Levine, R. A., \& Campbell, D. T. (1972). Theories of conflict, ethnic attitudes and group behavior. New York: John Willey \& Sons.

Levy, B. R., \& Banaji, M. R. (2002). Implicit ageism. In Ageism: Stereotyping and prejudice against older persons. (pp. 49-75). The MIT Press. 
Livingston, R. W., \& Brewer, M. B. (2002). What are we really priming? Cue-based versus category-based processing of facial stimuli. Journal of Personality and Social Psychology, 82(1), 5-18. https://doi.org/10.1037/0022-3514.82.1.5

Lundqvist, D., Flykt, A., \& Öhman, A. (1998). The Karolinska Directed Emotional Faces-KDEF, CD ROM from Department of Clinical Neuroscience, Psychology Section. Karolinska Institutet, 3-5.

Macrae, C. N., Bodenhausen, G. V., \& Milne, A. B. (1995). The dissection of selection in person perception: Inhibitory processes in social stereotyping. Journal of Personality and Social Psychology, 69(3), 397-407.

Macrae, C. N., Hewstone, M., \& Griffiths, R. J. (1993). Processing load and memory for stereotypebased information. European Journal of Social Psychology, 23(1), 77-87.

Macrae, C. N., Milne, A. B., \& Bodenhausen, G. V. (1994). Stereotypes as energy-saving devices: A peek inside the cognitive toolbox. Journal of Personality and Social Psychology, 66(1), $37-47$.

Makowski, D., Ben-Shachar, M. S., \& Lüdecke, D. (2019). BayestestR: Describing Effects and their Uncertainty, Existence and Significance within the Bayesian Framework. Journal of Open Source Software, 4(40), 1541. https://doi.org/10.21105/joss.01541

Mandelbaum, E. (2016). Attitude, inference, association: On the propositional structure of implicit bias. Noûs, 50(3), 629-658.

Martin, D., Hutchison, J., Slessor, G., Urquhart, J., Cunningham, S. J., \& Smith, K. (2014). The spontaneous formation of stereotypes via cumulative cultural evolution. Psychological Science, 25(9), 1777-1786. https://doi.org/10.1177/0956797614541129

McFarland, S. G., \& Crouch, Z. (2002). A cognitive skill confound on the Implicit Association Test. Social Cognition, 20(6), 483-510. 
Mierke, J., \& Klauer, K. C. (2003). Method-specific variance in the implicit association test. Journal of Personality and Social Psychology, 85(6), 1180-1192. https://doi.org/10.1037/0022-3514.85.6.1180

Monroe, B. M., Koenig, B. L., Wan, K. S., Laine, T., Gupta, S., \& Ortony, A. (2018). Reexamining dominance of categories in impression formation: A test of dual-process models. Journal of Personality and Social Psychology, 115(1), 1-30.

Moran, T., \& Bar-Anan, Y. (2013). The effect of object-valence relations on automatic evaluation. Cognition \& Emotion, 27(4), 743-752.

Moran, T., Bar-Anan, Y., \& Nosek, B. A. (2017). The effect of the validity of co-occurrence on automatic and deliberate evaluations: Validity and automatic evaluations. European Journal of Social Psychology, 47(6), 708-723. https://doi.org/10.1002/ejsp.2266

Mullen, B., Brown, R., \& Smith, C. (1992). Ingroup bias as a function of salience, relevance, and status: An integration. European Journal of Social Psychology, 22(2), 103-122. https://doi.org/10.1002/ejsp.2420220202

Navon, M. (2021, September). Discrepancies between automatic and deliberate evaluation of individual group-members. The ESCON Transfer of Knowledge Conference, Salzburg, Austria

Navon, M. \& Bar-Anan, Y. (2022). The relative effect of group-based information and individuating information on automatic judgment. Manuscript in preparation.

Navon, M., Shechter, A., \& Bar-Anan, Y. (2021). The effect of group membership and individuating information on automatic and deliberate evaluation of well-known people. Journal of Personality and Social Psychology, 121(3), 498-523.

Nosek, B. A. (2005). Moderators of the relationship between implicit and explicit evaluation. Journal of Experimental Psychology: General, 134(4), 565-584. https://doi.org/10.1037/0096-3445.134.4.565 
Nosek, B. A., Banaji, M., \& Greenwald, A. G. (2002). Harvesting implicit group attitudes and beliefs from a demonstration web site. Group Dynamics: Theory, Research, and Practice, 6(1), 101-115. https://doi.org/10.1037//1089-2699.6.1.101

Nosek, B. A., Greenwald, A. G., \& Banaji, M. R. (2007). The Implicit Association Test at age 7: A methodological and conceptual Review. In Social psychology and the unconscious: The automaticity of higher mental processes. (pp. 265-292). Psychology Press.

Nosek, B. A., Hawkins, C. B., \& Frazier, R. S. (2011). Implicit social cognition: From measures to mechanisms. Trends in Cognitive Sciences, 15(4), 152-159.

Nosek, B. A., Smyth, F. L., Hansen, J. J., Devos, T., Lindner, N. M., Ranganath, K. A., Smith, C. T., Olson, K. R., Chugh, D., Greenwald, A. G., \& Banaji, M. R. (2007). Pervasiveness and correlates of implicit attitudes and stereotypes. European Review of Social Psychology, 18(1), 36-88. https://doi.org/10.1080/10463280701489053

Oakes, P. J., Haslam, S. A., \& Turner, J. C. (1994). Stereotyping and social reality. Blackwell Publishing.

Oakes, P., \& Turner, J. (1980). Social categorization and intergroup behaviour: Does minimal intergroup discrimination make social identity more positive? European Journal of Social Psychology, 10(3), 295-301.

Olson, M. A., \& Fazio, R. H. (2004). Reducing the influence of extrapersonal associations on the Implicit Association Test: Personalizing the IAT. Journal of Personality and Social Psychology, 86(5), 653-667. https://doi.org/10.1037/0022-3514.86.5.653

Ottaway, S. A., Hayden, D. C., \& Oakes, M. A. (2001). Implicit attitudes and racism: Effects of word familiarity and frequency on the implicit association test. Social Cognition, 19(2), 97144. https://doi.org/10.1521/soco.19.2.97.20706

Paint.Net (4.2.13). (2020). [Computer software]. https://www.getpaint.net/download.html 
Park, J. H., Schaller, M., \& Van Vugt, M. (2008). Psychology of human kin recognition: Heuristic cues, erroneous inferences, and their implications. Review of General Psychology, 12(3), $215-235$.

Payne, B. K. (2001). Prejudice and perception: The role of automatic and controlled processes in misperceiving a weapon. Journal of Personality and Social Psychology, 81(2), 181-192. https://doi.org/10.1037/0022-3514.81.2.181

Payne, B. K., Cheng, C. M., Govorun, O., \& Stewart, B. D. (2005). An inkblot for attitudes: Affect misattribution as implicit measurement. Journal of Personality and Social Psychology, 89(3), 277.

Payne, B. K., Krosnick, J. A., Pasek, J., Lelkes, Y., Akhtar, O., \& Tompson, T. (2010). Implicit and explicit prejudice in the 2008 American presidential election. Journal of Experimental Social Psychology, 46(2), 367-374.

Petty, R. E., Briñol, P., \& DeMarree, K. G. (2007). The Meta-Cognitive Model (MCM) of attitudes: Implications for attitude measurement, change, and strength. Social Cognition, 25(5), 657686. https://doi.org/10.1521/soco.2007.25.5.657

Phelps, E. A., O’Connor, K. J., Cunningham, W. A., Funayama, E. S., Gatenby, J. C., Gore, J. C., \& Banaji, M. R. (2000). Performance on indirect measures of race evaluation predicts amygdala activation. Journal of Cognitive Neuroscience, 12(5), 729-738. https://doi.org/10.1162/089892900562552

Pinter, B., \& Greenwald, A. G. (2011). A comparison of minimal group induction procedures. Group Processes \& Intergroup Relations, 14(1), 81-98.

Plant, E. A., \& Devine, P. G. (1998). Internal and external motivation to respond without prejudice. Journal of Personality and Social Psychology, 75(3), 811-832. https://doi.org/10.1037/0022-3514.75.3.811 
Quinn, K. A., Mason, M. F., \& Macrae, C. N. (2010). When Arnold is “The Terminator”, We no longer see him as a man: The temporal determinants of person perception. Experimental Psychology, 57(1), 27-35. https://doi.org/10.1027/1618-3169/a000004

Ranganath, K. A., \& Nosek, B. A. (2008). Implicit attitude generalization occurs immediately; explicit attitude generalization takes time. Psychological Science, 19(3), 249-254. https://doi.org/10.1111/j.1467-9280.2008.02076.x

Ratner, K. G., Dotsch, R., Wigboldus, D. H., van Knippenberg, A., \& Amodio, D. M. (2014). Visualizing minimal ingroup and outgroup faces: Implications for impressions, attitudes, and behavior. Journal of Personality and Social Psychology, 106(6), 897-911. https://psycnet.apa.org/doi/10.1037/a0036498

Rothbart, M., Fulero, S., Jensen, C., Howard, J., \& Birrell, P. (1978). From individual to group impressions: Availability heuristics in stereotype formation. Journal of Experimental Social Psychology, 14(3), 237-255.

Rothermund, K., \& Wentura, D. (2001). Figure-ground asymmetries in the Implicit Association Test (IAT). Experimental Psychology, 48(2), 94-106. https://doi.org/10.1026//09493946.48.2.94

Rothermund, K., \& Wentura, D. (2004). Underlying processes in the Implicit Association Test: Dissociating salience from associations. Journal of Experimental Psychology: General, 133(2), 139-165. https://doi.org/10.1037/0096-3445.133.2.139

Rubinstein, R. S., Jussim, L., \& Stevens, S. T. (2018). Reliance on individuating information and stereotypes in implicit and explicit person perception. Journal of Experimental Social Psychology, 75, 54-70. https://doi.org/10.1016/j.jesp.2017.11.009

Sagarin, B. J., Ambler, J. K., \& Lee, E. M. (2014). An ethical approach to peeking at data. Perspectives on Psychological Science, 9(3), 293-304. 
Schmitz, F., Teige-Mocigemba, S., Voss, A., \& Klauer, K. C. (2013). When scoring algorithms matter: Effects of working memory load on different IAT scores. British Journal of Social Psychology, 52(1), 103-121.

Sidanius, J., \& Pratto, F. (1999). Social dominance: A theory of group-based inequality and oppression. New York, NY: Cambridge.

Silge, J., Chow, F., Kuhn, M., \& Wickham, H. (2021). rsample: General Resampling Infrastructure. https://CRAN.R-project.org/package=rsample

Spears, R., Doosje, B., \& Ellemers, N. (1997). Self-stereotyping in the face of threats to group status and distinctiveness: The role of group identification. Personality and Social Psychology Bulletin, 23(5), 538-553.

Stangor, C., \& Ford, T. E. (1992). Accuracy and expectancy-confirming processing orientations and the development of stereotypes and prejudice. European Review of Social Psychology, 3(1), $57-89$.

Stangor, C., Lynch, L., Duan, C., \& Glass, B. (1992). Categorization of individuals on the basis of multiple social features. Journal of Personality and Social Psycholog, 62(2), 207-218.

Stangor, C., \& McMillan, D. (1992). Memory for expectancy-congruent and expectancyincongruent information: A review of the social and social developmental literatures. Psychological Bulletin, 111(1), 42-61.

Steffens, M. C. (2004a). Is the implicit association test immune to faking? Experimental Psychology, 51(3), 165-179.

Steffens, M. C. (2004b). Is the Implicit Association Test Immune to Faking? Experimental Psychology, 51(3), 165-179. https://doi.org/10.1027/1618-3169.51.3.165

Stern, C., \& Axt, J. R. (2019). Group status modulates the associative strength between status quo supporting beliefs and anti-black attitudes. Social Psychological and Personality Science, 10(7), 946-956.

Tajfel, H. (1970). Experiments in intergroup discrimination. Scientific American, 223(5), 96-103. 
Tajfel, H. (1982). Social psychology of intergroup relations. Annual Review of Psychology, 33(1), $1-39$.

Tajfel, H., Turner, J. C., Austin, W. G., \& Worchel, S. (1979). An integrative theory of intergroup conflict. Organizational Identity: A Reader, 56(65), 9780203505984-16.

Teige-Mocigemba, S., \& Klauer, K. C. (2008). 'Automatic'evaluation? Strategic effects on affective priming. Journal of Experimental Social Psychology, 44(5), 1414-1417.

Thibault, J., \& Walker, L. (1975). Procedural justice: A social psychological analysis. Hillsdale, NJ: Lawrence Elbaum Associates.

Turner, J. C. (1975). Social comparison and social identity: Some prospects for intergroup behaviour. European Journal of Social Psychology, 5(1), 1-34.

Uhlmann, E. L., Brescoll, V. L., \& Paluck, E. L. (2006). Are members of low status groups perceived as bad, or badly off? Egalitarian negative associations and automatic prejudice. Journal of Experimental Social Psychology, 42(4), 491-499. https://doi.org/10.1016/j.jesp.2004.10.003

Unkelbach, C., \& Fiedler, K. (2020). The challenge of diagnostic inferences from implicit measures: The case of non-evaluative influences in the evaluative priming paradigm. Social Cognition, 38(Supplement), s208-s222.

Van Bavel, J. J., Packer, D. J., \& Cunningham, W. A. (2011). Modulation of the fusiform face area following minimal exposure to motivationally relevant faces: Evidence of in-group enhancement (not out-group disregard). Journal of Cognitive Neuroscience, 23(11), 33433354.

Van Dessel, P., Gawronski, B., \& De Houwer, J. (2019). Does explaining social behavior require multiple memory systems? Trends in Cognitive Sciences, 23(5), 368-369. https://doi.org/10.1016/j.tics.2019.02.001 
Van Dessel, P., Ratliff, K., Brannon, S. M., Gawronski, B., \& De Houwer, J. (2020). IllusoryCorrelation Effects on Implicit and Explicit Evaluation. Personality and Social Psychology Bulletin, 1-15.

Van Vugt, M., \& Schaller, M. (2008). Evolutionary approaches to group dynamics: An introduction. Group Dynamics: Theory, Research, and Practice, 12(1), 1-6.

Vianello, M., \& Bar-Anan, Y. (2021). Can the Implicit Association Test measure automatic judgment? The validation continues. Perspectives on Psychological Science, 16(2), 415421.

Von Hippel, W., Jonides, J., Hilton, J. L., \& Narayan, S. (1993). Inhibitory effect of schematic processing on perceptual encoding. Journal of Personality and Social Psychology, 64(6), 921.

Westfall, P. H., Johnson, W. O., \& Utts, J. M. (1997). A Bayesian perspective on the Bonferroni adjustment. Biometrika, 84(2), 419-427.

Wiers, R. W., Beckers, L., Houben, K., \& Hofmann, W. (2009). A short fuse after alcohol: Implicit power associations predict aggressiveness after alcohol consumption in young heavy drinkers with limited executive control. Pharmacology Biochemistry and Behavior, 93(3), $300-305$.

Wittenbrink, B., Judd, C., \& Park, B. (2001). Spontaneous prejudice in context: Variability in automatically activated attitudes. Journal of Personality and Social Psychology, 81(5), 815827.

Yamagishi, T., Jin, N., \& Kiyonari, T. (1999). Bounded generalized reciprocity: Ingroup boasting and ingroup favoritism. Advances in Group Processes, 16(1), 161-197. 